Article

\title{
Synthesis and Fungicidal Activities of (Z/E)-3,7-Dimethyl-2,6-octadienamide and Its 6,7-Epoxy Analogues
}

\author{
Mingyan Yang, Hongbo Dong, Jiazhen Jiang and Mingan Wang * \\ Received: 10 October 2015; Accepted: 17 November 2015; Published: 25 November 2015 \\ Academic Editor: Jean Jacques Vanden Eynde \\ Department of Applied Chemistry, China Agricultural University, Beijing 100193, China; \\ yangmy@cau.edu.cn (M.Y.); dhb1986115@cau.edu.cn (H.D.); jiangjiazhen@cau.edu.cn (J.J.) \\ * Correspondence: wangma@cau.edu.cn; Tel.: +86-10-6273-4093
}

\begin{abstract}
In order to find new lead compounds with high fungicidal activity, (Z/E)-3,7-dimethyl2,6-octadienoic acids were synthesized via selective two-step oxidation using the commercially available geraniol/nerol as raw materials. Twenty-eight different $(Z / E)$-3,7-dimethyl-2,6-octadienamide derivatives were prepared by reactions of $(Z / E)$-carboxylic acid with various aromatic and aliphatic amines, followed by oxidation of peroxyacetic acid to afford their 6,7-epoxy analogues. All of the compounds were characterized by HR-ESI-MS and ${ }^{1} \mathrm{H}-\mathrm{NMR}$ spectral data. The preliminary bioassays showed that some of these compounds exhibited good fungicidal activities against Rhizoctonia solani (R. solani) at a concentration of $50 \mu \mathrm{g} / \mathrm{mL}$. For example, 5C, $5 \mathbf{I}$ and $\mathbf{6 b}$ had $94.0 \%$, $93.4 \%$ and $91.5 \%$ inhibition rates against $R$. solani, respectively. Compound $5 \mathbf{f}$ displayed $\mathrm{EC}_{50}$ values of 4.3 and $9.7 \mu \mathrm{M}$ against Fusahum graminearum and R. Solani, respectively.
\end{abstract}

Keywords: 3,7-dimethyl-2,6-octadienamide; 3,7-dmethyl-6,7-epoxy-2-octadienamide; synthesis; fungicidal activity

\section{Introduction}

Amide compounds were widely used in pharmaceutical and agrochemical fields due to their wide range of biological activity. In pharmaceutical chemistry, some amides showed potent antibacterial activities and antiproliferative against human cancer cell lines including the drug-resistant cancer cells [1-3]. The other amides not only induce a significant decrease of antibiotic resistance in Gram-negative bacteria [4], but also exhibit antimicrobial activity against Staphylococcus aureus and Bacillus subtilis [5-7]. In agricultural chemicals, a lot of novel amide derivatives have been synthesized, some of them showed good fungicidal or insecticidal activities, and the mode of action on amide fungicides has been reviewed recently [8-16]. 2,6-Dimethyl-6-hydroxy-2E,4E-hepta-2, 4-diene acid and (6R)-3,7-dimethyl-7-hydroxyl-2-octen-1,6-olide were isolated from the fruit of Litsea cubeba in Tibet, and they were evaluated to have good fungicidal activities in our laboratory $[17,18]$. Based on these results, some of the seven-membered lactone derivatives were synthesized and confirmed to exhibit moderate to excellent fungicidal activities [19-21]. To the best of our knowledge, the biological activities of monoterpene acid amides were seldom paid attention. In order to find some novel derivatives with excellent fungicidal activity and explore the differences between 3,7-dimethyl-2,6-octadienoic acid and 3,7-dimethyl-7-hydroxyl-2-octen-1,6-olide (Scheme 1) against phytopathogens, $(Z / E)$-3,7-dimethyl-2,6-octadienoic acids were synthesized via two-step selective oxidation with the commercial available nerol/geraniol as the starting material $[19,22,23]$. Then, 28 different $(Z / E)$-3,7-dimethyl-2,6-octadienamide derivatives were designed and synthesized by reaction of the acid chloride and various aromatic and aliphatic amines [24], and their 6,7-epoxy 
derivatives were further obtained by epoxidation of the double bond between C-6 and C-7 [25]. The fungicidal activities of these amides and their 6,7-epoxy analogues against Fusahum graminearum, Rhizoctonia solani, Alternaria solani, Sclerotinia sclerotiorum, and Botrytis cinerea were evaluated. The synthetic route is shown as below (Scheme 2).

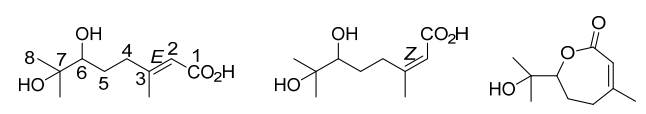

Scheme 1. Structures of (Z/E)-3,7-dimethyl-2,6-octadienoic acid and its 1,6-olide.

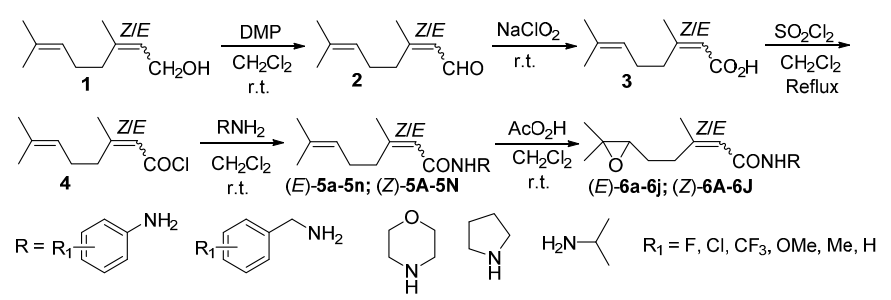

Scheme 2. Synthetic route of (Z/E)-3,7-dimethyl-2,6-octadienamide and their 6,7-epoxy analogues.

\section{Results and Discussion}

As we know, (Z)-3,7-dimethyl-2,6-octadienoic acid and (6R)-3,7-dimethyl-7-hydroxyl-2-octen-1,6-olide exhibited certain fungicidal activities [17,18]. The racemic 3,7-dimethyl-7-hydroxyl-2-octen-1,6-olide, the other seven-membered lactone derivatives and (E)-3,7-dimethyl-2,6-octadienoic acid were synthesized and some of them were found to exhibit better fungicidal activities in our laboratory [19-21]. In the total synthesis, we found that (E)-6,7-dihydroxy-3,7-dimethyl-2,6-octadienoic acid is an unstable compound because it is easily dehydrated under acid condition that needs to be purified rapidly and kept in low temperature. In this case, the 6,7-epoxy moiety was designed to replace the 6,7-dihydroxy group and keep the stability of the compound. Then 3,7-dimethyl-6,7-epoxy-2-octadienamides were designed and synthesized to observe the effect of epoxy moiety on the fungicidal activities. In consideration of the instability of epoxy moiety in strong acid condition, the route strategy of epoxidation-amidation was abandoned due to low product yields, the approach of amidation-epoxidation was selected to avoid the side reaction after optimizing repeatedly the reaction condition. The key intermediates $(Z / E)$-3,7-dimethyl-2,6-octadienoic acid were easily prepared in $85 \%$ and $76 \%$ yields over two steps with the Dess-Martin oxidant and Pinnick oxidation utilizing the commercially available nerol/geraniol as raw material [19]. Finally, the amides and the 6,7-epoxy amide derivatives were afforded in 15\%-79\% yields and $63 \%-96 \%$ yields under the mild condition, respectively.

In the ${ }^{1} \mathrm{H}-\mathrm{NMR}$ of compounds $5 \mathbf{a}-\mathbf{5 n}$, the olefin protons on $\mathrm{C}-2$ exhibited a singlet with the chemical shifts $\delta 5.47-5.77$, the olefin protons on C-6 displayed a multiplet at $\delta 4.90-5.20$ due to the coupling with the adjacent methylene protons at $\mathrm{C}-5$ and long range coupling with $\mathrm{CH}_{3}$ at $\mathrm{C}-7$. The methyls on C-3 had a doublet at $\delta 2.23-2.05$ with the coupling constant $1.2 \mathrm{~Hz}$ due to the long range coupling with the proton on $\mathrm{C}-2$, and the amide protons had the broad singlet with the chemical shifts $\delta 5.27-7.57$. While for the cis isomers $\mathbf{5 A}-\mathbf{5 N}$, the amide protons had the similar chemical shifts as the trans isomers, but the chemical shift of the protons on C-2 and C- 6 shifted to the downfield about $\delta$ $0.02-0.10$, and the methyls on C-3 shifted to the upfield about $\delta 0.30-0.40$.

In the ${ }^{1} \mathrm{H}$-NMR of 6,7-epoxy compounds $\mathbf{6 a - 6 j}$, the protons on $\mathrm{C}-2$, the amide protons and the methyls on $\mathrm{C}-3$ also showed the similar chemical shifts and coupling constants as that of compounds 5a-5n. However, the protons on C-6 had the chemical shifts $\delta 2.53-2.76$ due to existence of 6,7-epoxy group and the peaks split into the double of doublet due to the coupling with the two protons at C-5 with the coupling constants 5.5 and $7.0 \mathrm{~Hz}$, the chemical shifts of the two methyls at C-7 shifted to upfield about $\delta 0.30-0.35$. Similarly, for the cis isomers $6 \mathbf{A}-\mathbf{6 J}$, the protons on $\mathrm{C}-2$, the protons on 
C-6, the amide protons, the methyls on C-3, and the two methyls at C-7 had the similar chemical shifts and coupling constants. All of the new compounds were also characterized by HR-ESI-MS, and the $[\mathrm{M}+\mathrm{H}]^{+}$peaks were detected; their exact mass numbers matched well with the calculated molecule weights.

Based on the data in Table 1, compounds 5 and $\mathbf{6}$ showed a broad-spectrum of fungicidal activities against five tested agriculturally important phytopathgens; they were found to be particularly active against Rhizoctonia solani and Alternaria solani, for example, 5C, 5I and $\mathbf{6 b}$, respectively, exhibited $94.0 \%, 93.4 \%$ and $91.5 \%$ inhibition rates against $R$. solani, while $\mathbf{5 a}, \mathbf{5 c}, \mathbf{5 d}, \mathbf{5 g}, \mathbf{5 I}$ and $5 \mathrm{M}$ showed $86.4 \%, 86.0 \%, 88.9 \%, 88.7 \%, 89.5 \%$, and $85.5 \%$ inhibition rates against $A$. solani at the concentration of $50 \mu \mathrm{g} / \mathrm{mL}$, respectively. Greater than $70 \%$ inhibition at $50 \mu \mathrm{g} / \mathrm{mL}$ was considered to be good in terms of antifungal inhibition, greater than $90 \%$ excellent in this paper.

In the $Z / E$-amides, comparison of the inhibition rates of $5 \mathrm{l}, 5 \mathrm{~m}, 5 \mathrm{n}, 5 \mathrm{~L}, 5 \mathrm{M}$ and $5 \mathrm{~N}$ with $5 \mathbf{a}-5 \mathbf{k}$ and $\mathbf{5 A}-\mathbf{5 K}$, we found that the aromatic amides showed much better fungicidal activities than the aliphatic amides against $R$. solani and A. solani. It seemed that the aromatic substituted group contributed a lot to the fungicidal activities. Thus, the different aromatic groups such as phenyl, substituted phenyl, benzyl and substituted benzyl groups were selected to optimize the structure. By comparing the inhibition rates of compounds $5 b-5 g, 5 B-5 G$ with compound $5 a$ and $5 \mathrm{~A}$, we found that the ortho-substitution $(\mathrm{Cl}, \mathrm{F})$ was beneficial to improve the fungicidal activities such as $\mathbf{5 c}, \mathbf{5 d}, \mathbf{5 C}$ and $\mathbf{5 D}$, while the activities at the para-substitution were kept or reduced such as $\mathbf{5 b}, \mathbf{5 e}, \mathbf{5 f}, \mathbf{5 B}, \mathbf{5 e}$ and $\mathbf{5 F}$. However, one more $\mathrm{N}$-methyl group did not significantly change the activity comparing $\mathbf{5 a}$ and $5 \mathrm{~A}$ with $5 \mathrm{~g}$ and $5 \mathrm{G}$. So we concluded that the para-substitution was not helpful to improve the activity, especially the electron-withdraw substitution groups. Further, the amides with benzyl and substituted benzyl groups (compounds $5 \mathbf{h}-\mathbf{5} \mathbf{k}$ and $\mathbf{5} \mathbf{H}-\mathbf{5 K}$ ) were synthesized and assayed. The results in Table 1 indicated that amides with (substituted) benzyl groups had similar or increased fungicidal activities against $R$. solani and $A$. solani comparing with compound $5 \mathbf{a}$ and $5 \mathbf{A}$. Thus, the (substituted) benzyl groups have similar effects on the fungicidal activities as the (substituted) phenyl groups. The effect of the double bond configuration at $C_{2}$ and $C_{3}$ on the inhibition rates did not indicate significant differences by comparison the inhibition data of $5 \mathrm{a}-5 \mathrm{n}$ and $5 \mathrm{~A}-5 \mathrm{~N}$.

From the data in Table 1, it was very clear that compounds 5 showed much better fungicidal activities against all tested phytopathgens than compounds $\mathbf{6}$. While compounds $\mathbf{6 b}$ and $\mathbf{6 B}$ were two exceptions, which showed the inhibition rates of $91.5 \%$ and $82.7 \%$ against $R$. solani, respectively, much higher than the $59.5 \%$ and $55.4 \%$ inhibition rates of $5 \mathbf{b}$ and $5 \mathbf{B}$. The similar effects were observed for the aromatic and aliphatic amides, the substitution on the benzene ring of phenyl and benzyl groups, and the configuration of the double bond at $C_{2}$ and $C_{3}$. The double bond at $C_{6}$ and $C_{7}$ or adjacent 6,7-dihydroxy played an essential role for a better fungicidal activity when comparison the inhibition rate data of compounds 5 and $\mathbf{6}$.

Based on the above results, the $\mathrm{EC}_{50}$ values $\left(\mathrm{EC}_{50}\right.$ is the concentration of inhibition $50 \%$ fungus growth at tested condition.) were determined further for these compounds with more than $70 \%$ inhibition rates. The typical inhibition rates changing with the concentration could be seen in Figure 1. The data in Table 2 confirmed that most of compounds exhibited an inhibition against $R$. solani and A. solani with $\mathrm{EC}_{50}$ values between 9.7 and $677.8 \mu \mathrm{M}$, and several compounds were active against F. graminearum, S. sclerotiorum and B. cinerea with $\mathrm{EC}_{50}$ values between 4.3 and $92.9 \mu \mathrm{M}$. Among them, compound $5 \mathrm{f}$ had the best fungicidal activities with $\mathrm{EC}_{50}$ values of 4.3 and $9.7 \mu \mathrm{M}$ against F. graminearum and $R$. solani, respectively, and compounds $\mathbf{5 g}$ and $\mathbf{5 I}$ had the broad-spectrum of fungicidal activities against four phytopathgens with $\mathrm{EC}_{50}$ values between 17.1 and $61.2 \mu \mathrm{M}$, most of the other compounds have $\mathrm{EC}_{50}$ values between 13.4 and $97.9 \mu \mathrm{M}$ against $R$. solani and A. solani except $\mathbf{6 d}, \mathbf{6 f}, \mathbf{5 M}$ and $\mathbf{6 H}$. These results indicated that there would be the possible improvement of fungicidal activities against $R$. solani and A. solani if the chemical structures were further modified, especially on the structures of $\mathbf{5 f}, \mathbf{5 g}$ and $\mathbf{5 I}$. Optimizations on the aromatic amine moieties around compound 5 are in progress. 
Table 1. The fungicidal activities (inhibition rate, $\%$ ) of compounds 5 and 6 at $50 \mu \mathrm{g} / \mathrm{mL}$ a

\begin{tabular}{|c|c|c|c|c|c|c|c|c|c|c|c|c|c|}
\hline Compd. & $\mathbf{R}$ & F. $G$ & R. $S$ & A. $S$ & S. $S$ & B. $C$ & Compd. & $\mathbf{R}$ & F. $G$ & R. $S$ & A. $S$ & S. S & B. $C$ \\
\hline $5 a$ & $\mathrm{Ph}$ & 68.1 & 88.9 & 86.4 & 62.9 & 60.2 & $5 A$ & $\mathrm{Ph}$ & 63.7 & 79.9 & 85.0 & 72.3 & 66.7 \\
\hline $5 b$ & $2,4-\mathrm{Cl}_{2} \mathrm{Ph}$ & 17.7 & 59.5 & 77.6 & 36.2 & 22.9 & $5 B$ & $2,4-\mathrm{Cl}_{2} \mathrm{Ph}$ & 47.2 & 55.4 & 51.3 & 56.5 & 4.6 \\
\hline $5 c$ & $2-\mathrm{ClPh}$ & 51.9 & 85.3 & 86.0 & 57.4 & 44.4 & $5 C$ & 2-ClPh & 52.2 & 94.0 & 81.3 & 63.4 & 44.5 \\
\hline $5 d$ & $2-\mathrm{FPh}$ & 74.3 & 84.3 & 88.9 & 47.9 & 48.0 & $5 \mathrm{D}$ & $2-\mathrm{FPh}$ & 55.2 & 82.3 & 82.4 & 45.3 & 43.5 \\
\hline $5 e$ & $4-\mathrm{CF}_{3} \mathrm{Ph}$ & 73.4 & 65.6 & 63.9 & 23.4 & 26.8 & $5 \mathrm{E}$ & $4-\mathrm{CF}_{3} \mathrm{Ph}$ & 55.0 & 83.8 & 70.5 & 52.5 & 31.2 \\
\hline $5 f$ & $4-\mathrm{CH}_{3} \mathrm{Ph}$ & 77.0 & 86.0 & 77.1 & 53.6 & 26.5 & $5 \mathrm{~F}$ & $4-\mathrm{CH}_{3} \mathrm{Ph}$ & 46.7 & 71.4 & 74.4 & 70.4 & 42.7 \\
\hline $5 g$ & $\mathrm{Ph}, \mathrm{CH}_{3}$ & 50.6 & 86.7 & 88.7 & 77.1 & 83.2 & $5 G$ & $\mathrm{Ph}, \mathrm{CH}_{3}$ & 71.3 & 86.4 & 85.1 & 69.2 & 65.4 \\
\hline $5 \mathrm{~h}$ & $\mathrm{PhCH}_{2}$ & 55.5 & 86.7 & 72.4 & 34.6 & 51.0 & $5 \mathrm{H}$ & $\mathrm{PhCH}_{2}$ & 53.6 & 80.7 & 80.2 & 57.0 & 34.6 \\
\hline $5 i$ & $4-\mathrm{FPhCH}_{2}$ & 30.7 & 54.4 & 82.0 & 24.7 & 31.6 & $5 \mathrm{I}$ & 4-FPhCH 2 & 75.2 & 93.4 & 89.5 & 76.9 & 47.4 \\
\hline $5 j$ & $4-\mathrm{OCH}_{3} \mathrm{PhCH}_{2}$ & 45.3 & 83.0 & 78.9 & 46.6 & 64.0 & $5 \mathrm{~J}$ & $4-\mathrm{OCH}_{3} \mathrm{PhCH}_{2}$ & 52.5 & 77.6 & 81.6 & 46.9 & 19.1 \\
\hline $5 k$ & $2-\mathrm{ClPhCH}_{2}$ & 44.5 & 79.9 & 81.1 & 69.5 & 64.6 & $5 K$ & $2-\mathrm{ClPhCH}_{2}$ & 56.6 & 89.3 & 83.3 & 69.7 & 51.0 \\
\hline 51 & morpholino & 16.4 & 44.8 & 50.9 & 40.7 & 17.8 & $5 \mathrm{~L}$ & morpholino & 30.1 & 44.9 & 62.2 & 14.2 & 7.3 \\
\hline $5 \mathrm{~m}$ & pyrrolidin-1-yl & 39.7 & 60.0 & 79.8 & 28.1 & 29.2 & $5 M$ & pyrrolidin-1-yl & 63.4 & 78.8 & 85.5 & 43.1 & 35.6 \\
\hline $5 n$ & isopropyl & 15.6 & 48.7 & 58.2 & 12.8 & 14.3 & $5 N$ & isopropyl & 46.2 & 50.3 & 43.2 & 43.1 & 26.0 \\
\hline $6 a$ & $\mathrm{Ph}$ & 39.1 & 23.2 & 38.0 & 19.2 & 16.9 & $6 \mathrm{~A}$ & $\mathrm{Ph}$ & 32.8 & 30.3 & 69.3 & 36.4 & 22.8 \\
\hline $6 b$ & $2,4-\mathrm{Cl}_{2} \mathrm{Ph}$ & 63.0 & 91.5 & 73.6 & 72.7 & 30.4 & $6 B$ & $2,4-\mathrm{Cl}_{2} \mathrm{Ph}$ & 46.9 & 82.6 & 81.1 & 43.7 & 39.1 \\
\hline $6 c$ & $2-\mathrm{ClPh}$ & 39.8 & 35.6 & 59.1 & 33.3 & 13.5 & $6 C$ & $2-\mathrm{ClPh}$ & 33.2 & 30.4 & 15.4 & 33.9 & 31.6 \\
\hline $6 d$ & $4-\mathrm{CH}_{3} \mathrm{Ph}$ & 74.0 & 79.2 & 58.4 & 18.4 & 32.9 & $6 \mathrm{D}$ & $4-\mathrm{CH}_{3} \mathrm{Ph}$ & 46.7 & 43.9 & 73.4 & 3.72 & 2.50 \\
\hline $6 e$ & $\mathrm{Ph}, \mathrm{CH}_{3}$ & 68.5 & 59.1 & 66.5 & 46.2 & 10.7 & $6 \mathrm{E}$ & $\mathrm{Ph}, \mathrm{CH}_{3}$ & 36.2 & 62.6 & 54.2 & 0.0 & 1.6 \\
\hline $6 f$ & $\mathrm{PhCH}_{2}$ & 65.0 & 57.7 & 71.3 & 0.0 & 9.7 & $6 \mathrm{~F}$ & $\mathrm{PhCH}_{2}$ & 54.3 & 76.8 & 57.8 & 31.7 & 19.8 \\
\hline $6 \mathrm{~g}$ & $4-\mathrm{FPhCH}_{2}$ & 17.7 & 68.4 & 29.1 & 0.0 & 15.1 & $6 G$ & $4-\mathrm{FPhCH}_{2}$ & 40.4 & 38.9 & 55.7 & 28.7 & 10.9 \\
\hline $6 \mathrm{~h}$ & morpholino & 68.6 & 52.5 & 43.3 & 38.7 & 33.2 & $6 \mathrm{H}$ & morpholino & 33.8 & 73.3 & 52.9 & 0.0 & 17.6 \\
\hline $6 i$ & pyrrolidin-1-yl & 9.2 & 37.8 & 66.3 & 19.9 & 0.0 & $6 I$ & pyrrolidin-1-yl & 59.3 & 48.1 & 76.3 & 0.0 & 12.1 \\
\hline $6 j$ & isopropyl & 22.6 & 18.0 & 65.4 & 8.4 & 15.2 & $6 \mathrm{~J}$ & isopropyl & 36.3 & 53.0 & 36.8 & 10.6 & 38.4 \\
\hline \multicolumn{2}{|c|}{ Carbendazim } & 100 & 100 & 100 & 81.0 & 4.2 & \multicolumn{2}{|c|}{ Chlorothalonil } & 95.8 & 99.9 & 100 & 100 & 100 \\
\hline
\end{tabular}

${ }^{a}$ F. G: Fusahum graminearum, R. S: Rhizoctonia solani, A. S: Alternaria solani, S. S: Sclerotinia sclerotiorum, B. C: Botrytis cinerea. The data are the mean measurements were calculated from the three replicates with $0 \pm 5 \%$ errors. 
Table 2. The $\mathrm{EC}_{50}(\mu \mathrm{M})$ values with $95 \%$ confidential interval in parenthesis of compounds 5 and $\mathbf{6}$ against different phytopathgens for the compounds with more than $70 \%$ inhibition rates in Table 1 .

\begin{tabular}{cccccc}
\hline Compd. & F. graminearum & R. solani & A. solani & S. sclerotiorum & B. cinerea \\
\hline 5a & - & $26.1(20.3-33.6)$ & $19.8(14.5-27.1)$ & - & - \\
5b & - & - & $51.0(45.3-57.4)$ & - & - \\
5c & - & $33.9(26.0-44.0)$ & $28.6(25.8-31.7)$ & - & - \\
5d & - & $19.2(12.7-25.6)$ & $39.8(28.6-48.6)$ & $202.7(155.0-263.1)$ & - \\
5e & $11.9(7.6-16.9)$ & - & - & - & - \\
5f & $4.3(3.2-5.8)$ & $9.7(25.1-56.2)$ & $37.6(45.6-59.5)$ & - & - \\
5g & - & $19.1(13.9-26.1)$ & $27.2(21.9-33.7)$ & $61.2(44.5-101.2)$ & $56.2(38.2-82.6)$ \\
5h & - & $30.4(24.5-37.6)$ & $27.6(21.3-35.7)$ & - & - \\
5i & - & - & $32.8(25.5-42.1)$ & - & - \\
5j & - & $35.5(25.2-44.4)$ & $27.8(23.3-29.6)$ & - & - \\
5k & - & $29.8(24.1-32.9)$ & $41.3(32.0-47.5)$ & - & - \\
5m & - & - & $50.3(33.3-75.7)$ & - & - \\
6b & - & $62.3(44.9-86.6)$ & $43.4(31.1-60.5)$ & $21.6(13.8-33.5)$ & - \\
6d & $57.4(52.7-62.3)$ & $130.7(92.1-185.1)$ & - & - & - \\
6f & - & - & $189.4(155.4-230.4)$ & - & - \\
5A & - & $97.9(88.3-108.4)$ & $14.0(11.5-17.1)$ & $92.9(67.9-126.9)$ & - \\
5C & - & $80.1(56.4-113.4)$ & $62.6(41.8-93.6)$ & - & - \\
5D & - & $54.5(35.6-73.5)$ & $26.0(19.7-30.2)$ & - & - \\
5E & - & $51.2(42.5-55.5)$ & $22.9(16.9-27.9)$ & - & - \\
5F & - & $39.0(29.5-51.4)$ & $42.6(30.6-59.0)$ & $23.2(16.8-31.9)$ & - \\
5G & - & $13.4(11.6-15.5)$ & $35.9(27.0-47.6)$ & $125.2(96.3-161.3)$ & - \\
5H & - & $41.4(26.8-63.6)$ & $21.2(16.3-27.4)$ & - & - \\
5I & $38.8(32.5-46.3)$ & $18.7(12.6-27.6)$ & $17.1(12.6-23.2)$ & $40.7(35.2-47.0)$ & - \\
5J & - & $57.9(38.9-76.7)$ & $31.1(23.5-36.6)$ & - & - \\
5K & - & $18.6(14.9-20.8)$ & $32.0(25.3-36.1)$ & - & - \\
5M & - & $284.1(207.7-387.9)$ & $63.8(42.6-95.3)$ & - & - \\
6B & - & $31.6(20.3-49.2)$ & $43.4(28.8-65.1)$ & - & - \\
6D & - & - & $667.3(462.3-999.5)$ & - & - \\
6F & - & $>1000$ & - & - & - \\
6H & - & $677.8(622.7-736.6)$ & - & - & - \\
6I & - & - & $83.8(56.9-123.1)$ & - & - \\
\hline & & - & & - & - \\
\end{tabular}

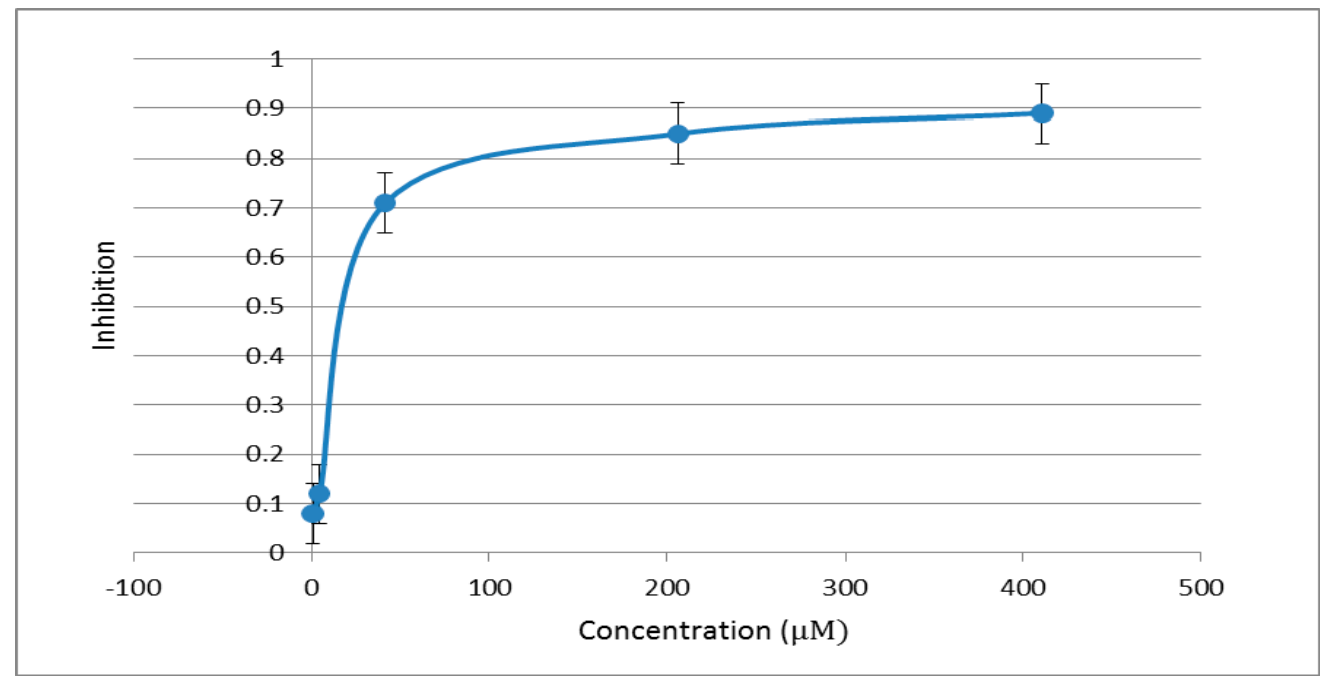

Figure 1. Inhibition rates of $\mathbf{5 a}$ against $A$. solani at different concentrations.

\section{Experimental Section}

\subsection{General Information}

All reactions were performed with magnetic stirring. Unless otherwise stated, all reagents were purchased from commercial suppliers and used without further purification. Organic solutions were concentrated under reduced pressure using a rotary evaporator or oil pump. Melting points 
were measured on a Yanagimoto apparatus (Yanagimoto MFG Co., Kyoto, Japan) and uncorrected. ${ }^{1}$ H-NMR spectra were obtained on Bruker DPX 300 spectrometer (Bruker Biospin Co., Stuttgart, Germany) with $\mathrm{CDCl}_{3}$ as a solvent and TMS as an internal standard. High-resolution mass spectral analysis was performed on a LTQ Orbitrap instrument (ThermoFisher scientific Inc., Waltham, MA, USA).

\subsection{Synthesis}

\subsubsection{Synthesis of (Z/E)-3,7-Dimethyl-2,6-octadienal (2, Neral and Geranial)}

According to the literature protocol [19,22], geraniol (8.0 g, $52 \mathrm{mmol})$, Dess-Martin Periodinane $(26.6 \mathrm{~g}, 63 \mathrm{mmol})$ and $320 \mathrm{~mL}$ DCM were added to a $500 \mathrm{~mL}$ three-necked flask at room temperature. The mixture was stirred for $4 \mathrm{~h}$ at the room temperature. Then the mixture was filtered and the filtrate was washed with saturated $\mathrm{NaHCO}_{3}$ solution, brine and dried over anhydrous $\mathrm{Na}_{2} \mathrm{SO}_{4}$. The solvent was removed under reduced pressure. The residue was purified by column chromatography using silica gel (petroleum ether/EtOAc 15:1) to give (E)-3,7-dimethyl-2,6-octadienal (7.9 g, 94\%) as a colorless oil; the (Z)-isomer (10.7 g, 88\%) was also prepared as a colorless oil using nerol (12.3 g) as starting material following the same procedure [26,27].

\subsubsection{Synthesis of (Z/E)-3,7-Dimethyl-2,6-octadienoic Acid (3, Geranic Acid and Nerolic Acid)}

According to the approaches in the literature [23], a solution of $\mathrm{NaClO}_{2}(22.0 \mathrm{~g}, 30 \mathrm{mmol})$ and $\mathrm{NaH}_{2} \mathrm{PO}_{4} \cdot 2 \mathrm{H}_{2} \mathrm{O}(34.4 \mathrm{~g}, 22 \mathrm{mmol})$ in water was added dropwise to a solution of (E)-3,7-dimethyl-2,6-octadienal ( $4.0 \mathrm{~g}, 22 \mathrm{mmol})$ and 2-methyl-2-butene in acetone $(220 \mathrm{~mL})$ at room temperature and stirred for $4 \mathrm{~h}$, the solution was extracted with ethyl acetate, and the organic layer was washed with brine, dried over anhydrous $\mathrm{Na}_{2} \mathrm{SO}_{4}$. The solvent was removed under reduced pressure and the residue was purified by column chromatography using silica gel (petroleum ether/EtOAc/ $\mathrm{CH}_{3} \mathrm{COOH}$ 25:1:0.5) to afford (E)-3,7-dimethyl-2,6-octadienoic acid (4.0 g, 90\%) as a colorless oil; the (Z)-isomer ( $8.5 \mathrm{~g}, 86 \%$ ) was also prepared as a colorless oil using (Z)-3,7-dimethyl2,6-octadienal (12.3 g) as the starting material following the same procedure [28].

\subsubsection{General Procedure for the Synthesis of Compounds 5}

Take compound $\mathbf{5 a}$ as an example: according to the procedure in the literature [22], $\mathrm{SOCl}_{2}$ $(1.4 \mathrm{~mL})$ was added to a solution of (E)-3,7-dimethyl-2,6-octadienoic acid $(0.8 \mathrm{~g}, 5 \mathrm{mmol})$ in $40 \mathrm{~mL}$ DCM in a $100 \mathrm{~mL}$ flask at room temperature. The mixture was stirred and heated at $40{ }^{\circ} \mathrm{C}$ for $4 \mathrm{~h}$. Then cool down to room temperature and remove the solvent under reduced pressure. The residue was dissolved in $10 \mathrm{~mL} \mathrm{DCM}$, the $10 \mathrm{~mL}$ DCM solution of aniline $(0.92 \mathrm{~mL}, 10 \mathrm{mmol})$ was added and stirred for $10 \mathrm{~h}$ at room temperature. Quenched the reaction with water, extracted with DCM, and the organic layer was washed with brine, dried over anhydrous $\mathrm{Na}_{2} \mathrm{SO}_{4}$. Removed the solvent under reduced pressure and the residue was purified by column chromatography to afford compound $\mathbf{5 a}$.

(E)-3,7-Dimethyl-N-phenyl-2,6-octadienamide (5a), grey oil, yield 79\%. ${ }^{1} \mathrm{H}-\mathrm{NMR}\left(300 \mathrm{MHz}, \mathrm{CDCl}_{3}\right) \delta$ : $7.53(\mathrm{~d}, J=7.9 \mathrm{~Hz}, 2 \mathrm{H}, \mathrm{ArH}), 7.34-7.29(\mathrm{~m}, 2 \mathrm{H}, \mathrm{ArH}), 7.11-7.06(\mathrm{~m}, 2 \mathrm{H}, \mathrm{ArH}+\mathrm{NH}), 5.69(\mathrm{~s}, 1 \mathrm{H},=\mathrm{CH})$, 5.14-5.05 (m, 1H, =CH), $2.22\left(\mathrm{~d}, J=1.2 \mathrm{~Hz}, 3 \mathrm{H}, \mathrm{CH}_{3}\right), 2.17$ (br.s, $\left.4 \mathrm{H}, 2 \times \mathrm{CH}_{2}\right), 1.70\left(\mathrm{~s}, 3 \mathrm{H}, \mathrm{CH}_{3}\right), 1.62$ (s, 3H, $\mathrm{CH}_{3}$ ); HR-MS (ESI) $m / z: \mathrm{C}_{16} \mathrm{H}_{21} \mathrm{NO}[\mathrm{M}+\mathrm{H}]^{+}$, Calcd. 244.1696; Found 244.1693. The spectral data were identical with those in the reference [29].

(E)-N-(2,4-Dichlorophenyl)-3,7-dimethyl-2,6-octadienamide (5b), pale yellow oil, yield 78\%. ${ }^{1} \mathrm{H}-\mathrm{NMR}$ $\left(300 \mathrm{MHz}, \mathrm{CDCl}_{3}\right) \delta: 8.44(\mathrm{~d}, J=8.9 \mathrm{~Hz}, 1 \mathrm{H}, \mathrm{ArH}), 7.50(\mathrm{~s}, 1 \mathrm{H}, \mathrm{NH}), 7.37(\mathrm{~d}, J=2.4 \mathrm{~Hz}, 1 \mathrm{H}, \mathrm{ArH}), 7.24$ $(\mathrm{dd}, J=8.9,2.4 \mathrm{~Hz}, 1 \mathrm{H}, \mathrm{ArH}), 5.73(\mathrm{~s}, 1 \mathrm{H},=\mathrm{CH}), 5.13-5.04(\mathrm{~m}, 1 \mathrm{H},=\mathrm{CH}), 2.23\left(\mathrm{~d}, J=1.2 \mathrm{~Hz}, 3 \mathrm{H}, \mathrm{CH}_{3}\right)$, 2.20-2.18 (m, 4H, $\left.2 \times \mathrm{CH}_{2}\right), 1.70\left(\mathrm{~s}, 3 \mathrm{H}, \mathrm{CH}_{3}\right), 1.63\left(\mathrm{~s}, 3 \mathrm{H}, \mathrm{CH}_{3}\right)$; HR-MS (ESI) $m / z: \mathrm{C}_{16} \mathrm{H}_{19} \mathrm{Cl}_{2} \mathrm{NO}$ $[\mathrm{M}+\mathrm{H}]^{+}$, Calcd. 312.0916; Found 312.0917. 
(E)-N-(2-Chlorophenyl)-3,7-dimethyl-2,6-octadienamide (5c), yellow oil, yield 69\%. ${ }^{1} \mathrm{H}-\mathrm{NMR}(300 \mathrm{MHz}$, $\left.\mathrm{CDCl}_{3}\right) \delta: 8.45(\mathrm{~d}, J=8.1 \mathrm{~Hz}, 1 \mathrm{H}, \mathrm{ArH}), 7.57(\mathrm{~s}, 1 \mathrm{H}, \mathrm{NH}), 7.35(\mathrm{dd}, J=8.1,1.5 \mathrm{~Hz}, 1 \mathrm{H}, \mathrm{ArH}), 7.30-7.23$ $(\mathrm{m}, 1 \mathrm{H}, \mathrm{ArH}), 7.04-6.98(\mathrm{~m}, 1 \mathrm{H}, \mathrm{ArH}), 5.75(\mathrm{~s}, 1 \mathrm{H},=\mathrm{CH}), 5.14-5.04(\mathrm{~m}, 1 \mathrm{H},=\mathrm{CH}), 2.23(\mathrm{~d}, J=1.2 \mathrm{~Hz}$, $\left.3 \mathrm{H}, \mathrm{CH}_{3}\right), 2.20$ (br.s, $\left.4 \mathrm{H}, 2 \times \mathrm{CH}_{2}\right), 1.71\left(\mathrm{~s}, 3 \mathrm{H}, \mathrm{CH}_{3}\right), 1.64\left(\mathrm{~s}, 3 \mathrm{H}, \mathrm{CH}_{3}\right) ; \mathrm{HR}-\mathrm{MS}$ (ESI) $m / z: \mathrm{C}_{16} \mathrm{H}_{20} \mathrm{ClNO}$ $[\mathrm{M}+\mathrm{H}]^{+}$, Calcd. 278.1306; Found 278.1303.

(E)-N-(2-Fluorophenyl)-3,7-dimethyl-2,6-octadienamide (5d), brown oil, yield 35\%. ${ }^{1} \mathrm{H}-\mathrm{NMR}(300 \mathrm{MHz}$, $\left.\mathrm{CDCl}_{3}\right)$ 8: 8.42-8.36 (m, 1H, ArH), $7.29(\mathrm{~s}, 1 \mathrm{H}, \mathrm{NH}), 7.15-6.99(\mathrm{~m}, 3 \mathrm{H}, \mathrm{ArH}), 5.73(\mathrm{~s}, 1 \mathrm{H},=\mathrm{CH})$, 5.16-5.09 (m, 1H, =CH), $2.23\left(\mathrm{~d}, J=1.2 \mathrm{~Hz}, 3 \mathrm{H}, \mathrm{CH}_{3}\right), 2.19$ (br.s, $\left.4 \mathrm{H}, 2 \times \mathrm{CH}_{2}\right), 1.70\left(\mathrm{~s}, 3 \mathrm{H}, \mathrm{CH}_{3}\right)$, $1.63\left(\mathrm{~s}, 3 \mathrm{H}, \mathrm{CH}_{3}\right) ; \mathrm{HR}-\mathrm{MS}$ (ESI) $m / z: \mathrm{C}_{16} \mathrm{H}_{20} \mathrm{FNO}[\mathrm{M}+\mathrm{H}]^{+}$, Calcd. 262.1601; Found 262.1600.

(E)-N-(4-Trifluoromethylphenyl)-3,7-dimethyl-2,6-octadienamide (5e), yellow oil, yield $16 \% .{ }^{1} \mathrm{H}-\mathrm{NMR}$ $\left(300 \mathrm{MHz}, \mathrm{CDCl}_{3}\right) \delta: 7.66(\mathrm{~d}, J=8.7 \mathrm{~Hz}, 2 \mathrm{H}, \mathrm{ArH}), 7.55(\mathrm{~d}, J=8.7 \mathrm{~Hz}, 2 \mathrm{H}, \mathrm{ArH}), 7.26(\mathrm{~s}, 1 \mathrm{H}, \mathrm{NH}), 5.70$ $(\mathrm{s}, 1 \mathrm{H},=\mathrm{CH}), 5.15-5.08(\mathrm{~m}, 1 \mathrm{H},=\mathrm{CH}), 2.23\left(\mathrm{~d}, J=1.2 \mathrm{~Hz}, 3 \mathrm{H}, \mathrm{CH}_{3}\right), 2.19$ (br.s, $\left.4 \mathrm{H}, 2 \times \mathrm{CH}_{2}\right), 1.70(\mathrm{~s}, 3 \mathrm{H}$, $\left.\mathrm{CH}_{3}\right), 1.62\left(\mathrm{~s}, 3 \mathrm{H}, \mathrm{CH}_{3}\right)$; HR-MS (ESI) $m / z: \mathrm{C}_{17} \mathrm{H}_{20} \mathrm{~F}_{3} \mathrm{NO}[\mathrm{M}+\mathrm{H}]^{+}$, Calcd. 312.1569; Found 312.1568.

(E)-N-(4-Methylphenyl)-3,7-dimethyl-2,6-octadienamide (5f), brown oil, yield 74\%. ${ }^{1} \mathrm{H}-\mathrm{NMR}(300 \mathrm{MHz}$, $\left.\mathrm{CDCl}_{3}\right) \delta: 7.42(\mathrm{~d}, J=7.5 \mathrm{~Hz}, 2 \mathrm{H}, \mathrm{ArH}), 7.11(\mathrm{~d}, J=7.5 \mathrm{~Hz}, 3 \mathrm{H}, \mathrm{ArH}+\mathrm{NH}), 5.68(\mathrm{~s}, 1 \mathrm{H},=\mathrm{CH}), 5.14-5.07$ $(\mathrm{m}, 1 \mathrm{H},=\mathrm{CH}), 2.31\left(\mathrm{~s}, 3 \mathrm{H}, \mathrm{ArCH}_{3}\right), 2.21\left(\mathrm{~d}, J=1.2 \mathrm{~Hz}, 3 \mathrm{H}, \mathrm{CH}_{3}\right), 2.18-2.15\left(\mathrm{~m}, 4 \mathrm{H}, 2 \times \mathrm{CH}_{2}\right), 1.70(\mathrm{~s}$, $\left.3 \mathrm{H}, \mathrm{CH}_{3}\right), 1.62\left(\mathrm{~s}, 3 \mathrm{H}, \mathrm{CH}_{3}\right)$; HR-MS (ESI) $m / z: \mathrm{C}_{17} \mathrm{H}_{23} \mathrm{NO}[\mathrm{M}+\mathrm{H}]^{+}$, Calcd. 258.1852; Found 258.1852.

(E)-N-Phenyl-N,3,7-trimethyl-2,6-octadienamide (5g), brown oil, yield 76\%. ${ }^{1} \mathrm{H}-\mathrm{NMR}\left(300 \mathrm{MHz}, \mathrm{CDCl}_{3}\right)$ ס: 7.40-7.34 (m, 2H, ArH), 7.30-7.24 (m, 1H, ArH), 7.18-7.14 (m, 2H, ArH), 5.47 (br.s, 1H, =CH), 4.96-4.91 (m, 1H, =CH), $3.32\left(\mathrm{~s}, 3 \mathrm{H}, \mathrm{NCH}_{3}\right), 2.10\left(\mathrm{~d}, J=1.2 \mathrm{~Hz}, 3 \mathrm{H}, \mathrm{CH}_{3}\right), 1.95$ (br.s, $\left.4 \mathrm{H}, 2 \times \mathrm{CH}_{2}\right)$, $1.63\left(\mathrm{~s}, 3 \mathrm{H}, \mathrm{CH}_{3}\right), 1.51\left(\mathrm{~s}, 3 \mathrm{H}, \mathrm{CH}_{3}\right)$; HR-MS (ESI) $m / z: \mathrm{C}_{17} \mathrm{H}_{23} \mathrm{NO}[\mathrm{M}+\mathrm{H}]^{+}$, Calcd. 258.1852; Found 258.1850. The spectral data were identical with those in the reference [30].

(E)-N-Benzyl-3,7-dimethyl-2,6-octadienamide (5h), yellow oil, yield 71\%. ${ }^{1} \mathrm{H}-\mathrm{NMR}\left(300 \mathrm{MHz}, \mathrm{CDCl}_{3}\right)$

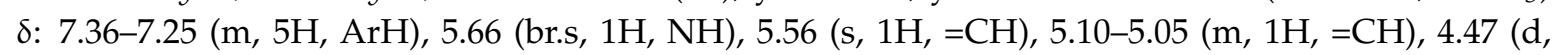
$\left.J=6.0 \mathrm{~Hz}, 2 \mathrm{H}, \mathrm{ArCH}_{2}\right), 2.18\left(\mathrm{~d}, J=1.2 \mathrm{~Hz}, 3 \mathrm{H}, \mathrm{CH}_{3}\right), 2.14-2.08\left(\mathrm{~m}, 4 \mathrm{H}, 2 \times \mathrm{CH}_{2}\right), 1.68\left(\mathrm{~s}, 3 \mathrm{H}, \mathrm{CH}_{3}\right)$, $1.60\left(\mathrm{~s}, 3 \mathrm{H}, \mathrm{CH}_{3}\right.$ ); HR-MS (ESI) $m / z: \mathrm{C}_{17} \mathrm{H}_{23} \mathrm{NO}[\mathrm{M}+\mathrm{H}]^{+}$, Calcd. 258.1852; Found 258.1850. The spectral data were identical with those in the reference [31].

(E)-N-(4-Fluorobenzyl)-3,7-dimethyl-2,6-octadienamide (5i), yellow oil, yield 73\%. ${ }^{1} \mathrm{H}-\mathrm{NMR}(300 \mathrm{MHz}$, $\left.\mathrm{CDCl}_{3}\right)$ 8: 7.30-7.23 (m, 2H, ArH), 7.05-6.98 (m, 2H, ArH), 5.64 (br.s, $\left.1 \mathrm{H}, \mathrm{NH}\right), 5.55$ (s, $\left.1 \mathrm{H},=\mathrm{CH}\right)$, 5.09-5.04 (m, 1H, =CH), $4.44\left(\mathrm{~d}, J=5.8 \mathrm{~Hz}, 2 \mathrm{H}, \mathrm{ArCH}_{2}\right), 2.18\left(\mathrm{~d}, J=1.2 \mathrm{~Hz}, 3 \mathrm{H}, \mathrm{CH}_{3}\right), 2.14-2.08(\mathrm{~m}$, $\left.4 \mathrm{H}, 2 \times \mathrm{CH}_{2}\right), 1.68\left(\mathrm{~s}, 3 \mathrm{H}, \mathrm{CH}_{3}\right), 1.60\left(\mathrm{~s}, 3 \mathrm{H}, \mathrm{CH}_{3}\right) ; \mathrm{HR}-\mathrm{MS}(\mathrm{ESI}) \mathrm{m} / z: \mathrm{C}_{17} \mathrm{H}_{22} \mathrm{FNO}[\mathrm{M}+\mathrm{H}]^{+}$: Calcd. 276.1758; Found 276.1754.

(E)-N-(4-Methoxybenzyl)-3,7-dimethyl-2,6-octadienamide (5j), yellow oil, yield 53\%. ${ }^{1} \mathrm{H}-\mathrm{NMR}(300 \mathrm{MHz}$, $\left.\mathrm{CDCl}_{3}\right) \delta: 7.23(\mathrm{~d}, J=8.7 \mathrm{~Hz}, 2 \mathrm{H}, \mathrm{ArH}), 6.86(\mathrm{~d}, J=8.7 \mathrm{~Hz}, 2 \mathrm{H}, \mathrm{ArH}), 5.57(\mathrm{~s}, 1 \mathrm{H}, \mathrm{NH}), 5.53(\mathrm{~s}, 1 \mathrm{H},=\mathrm{CH})$, $5.08-5.05(\mathrm{~m}, 1 \mathrm{H},=\mathrm{CH}), 4.41\left(\mathrm{~d}, J=6.0 \mathrm{~Hz}, 2 \mathrm{H}, \mathrm{ArCH}_{2}\right), 3.80\left(\mathrm{~s}, 3 \mathrm{H}, \mathrm{OCH}_{3}\right), 2.17(\mathrm{~d}, J=1.2 \mathrm{~Hz}, 3 \mathrm{H}$, $\left.\mathrm{CH}_{3}\right), 2.13-2.08\left(\mathrm{~m}, 4 \mathrm{H}, 2 \times \mathrm{CH}_{2}\right), 1.68\left(\mathrm{~s}, 3 \mathrm{H}, \mathrm{CH}_{3}\right), 1.60\left(\mathrm{~s}, 3 \mathrm{H}, \mathrm{CH}_{3}\right) ; \mathrm{HR}-\mathrm{MS}$ (ESI) $m / z: \mathrm{C}_{18} \mathrm{H}_{25} \mathrm{NO}_{2}$ $[\mathrm{M}+\mathrm{H}]^{+}$: Calcd. 288.1958; Found 288.1954.

(E)-N-(2-Chlorobenzyl)-3,7-dimethyl-2,6-octadienamide (5k), yellow oil, 45\%. ${ }^{1} \mathrm{H}-\mathrm{NMR}(300 \mathrm{MHz}$, $\left.\mathrm{CDCl}_{3}\right) \delta: 7.43-7.32(\mathrm{~m}, 2 \mathrm{H}, \mathrm{ArH}), 7.24-7.18(\mathrm{~m}, 2 \mathrm{H}, \mathrm{ArH}), 5.83(\mathrm{~s}, 1 \mathrm{H}, \mathrm{NH}), 5.57(\mathrm{~s}, 1 \mathrm{H},=\mathrm{CH})$, $5.08-5.04(\mathrm{~m}, 1 \mathrm{H},=\mathrm{CH}), 4.55\left(\mathrm{~d}, J=6.0 \mathrm{~Hz}, 2 \mathrm{H}, \mathrm{CH}_{2}\right), 2.15\left(\mathrm{~d}, J=1.2 \mathrm{~Hz}, 3 \mathrm{H}, \mathrm{CH}_{3}\right), 2.11$ (br.s, $4 \mathrm{H}$, $\left.2 \times \mathrm{CH}_{2}\right), 1.67\left(\mathrm{~s}, 3 \mathrm{H}, \mathrm{CH}_{3}\right), 1.59\left(\mathrm{~s}, 3 \mathrm{H}, \mathrm{CH}_{3}\right) ; \mathrm{HR}-\mathrm{MS}(\mathrm{ESI}) \mathrm{m} / z: \mathrm{C}_{17} \mathrm{H}_{22} \mathrm{ClNO}[\mathrm{M}+\mathrm{H}]^{+}$: Calcd. 292.1462; Found 292.1460.

(E)-3,7-Dimethyl-1-morpholino-2,6-octadien-1-one (51), yellow oil, yield 70\%. ${ }^{1} \mathrm{H}-\mathrm{NMR}(300 \mathrm{MHz}$, $\left.\mathrm{CDCl}_{3}\right) \delta: 5.72(\mathrm{~s}, 1 \mathrm{H},=\mathrm{CH}), 5.09-5.05(\mathrm{~m}, 1 \mathrm{H},=\mathrm{CH}), 3.67\left(\mathrm{br}, 6 \mathrm{H}, 3 \times \mathrm{CH}_{2}\right), 3.50\left(\mathrm{br}, 2 \mathrm{H}, \mathrm{CH}_{2}\right)$, 
2.17-2.13 (m, $\left.4 \mathrm{H}, 2 \times \mathrm{CH}_{2}\right), 1.88\left(\mathrm{~d}, J=1.2 \mathrm{~Hz}, 3 \mathrm{H}, \mathrm{CH}_{3}\right), 1.67\left(\mathrm{~s}, 3 \mathrm{H}, \mathrm{CH}_{3}\right), 1.61\left(\mathrm{~s}, 3 \mathrm{H}, \mathrm{CH}_{3}\right) ; \mathrm{HR}-\mathrm{MS}$ (ESI) $m / z: \mathrm{C}_{14} \mathrm{H}_{23} \mathrm{NO}_{2}[\mathrm{M}+\mathrm{H}]^{+}$, Calcd. 238.1800; Found 238.1801.

(E)-3,7-Dimethyl-1-(pyrrolidin-1-yl)-2,6-octadien-1-one (5m), colorless oil, yield 61\%. ${ }^{1} \mathrm{H}-\mathrm{NMR}(300 \mathrm{MHz}$, $\left.\mathrm{CDCl}_{3}\right) \delta: 5.77(\mathrm{~s}, 1 \mathrm{H},=\mathrm{CH}), 5.10-5.07(\mathrm{~m}, 1 \mathrm{H},=\mathrm{CH}), 3.50\left(\mathrm{t}, J=6.6 \mathrm{~Hz}, 2 \mathrm{H}, \mathrm{NCH}_{2}\right), 3.42(\mathrm{t}, J=6.6 \mathrm{~Hz}$, $\left.2 \mathrm{H}, \mathrm{NCH}_{2}\right), 2.18-2.12\left(\mathrm{~m}, 4 \mathrm{H}, 2 \times \mathrm{CH}_{2}\right), 2.05\left(\mathrm{~d}, J=1.2 \mathrm{~Hz}, 3 \mathrm{H}, \mathrm{CH}_{3}\right), 1.95-1.83\left(\mathrm{~m}, 4 \mathrm{H}, 2 \times \mathrm{CH}_{2}\right)$, $1.69\left(\mathrm{~s}, 3 \mathrm{H}, \mathrm{CH}_{3}\right), 1.61\left(\mathrm{~s}, 3 \mathrm{H}, \mathrm{CH}_{3}\right)$; HR-MS (ESI) $m / z: \mathrm{C}_{14} \mathrm{H}_{23} \mathrm{NO}[\mathrm{M}+\mathrm{H}]^{+}$, Calcd. 222.1852; Found 222.1849. The spectral data were identical with those in the reference [28].

(E)-N-Isopropyl-3,7-dimethyl-2,6-octadienamide (5n), yellow oil, yield 70\%. ${ }^{1} \mathrm{H}-\mathrm{NMR}\left(300 \mathrm{MHz}, \mathrm{CDCl}_{3}\right)$

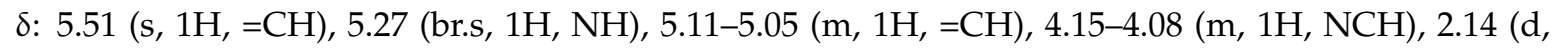
$\left.J=1.2 \mathrm{~Hz}, 3 \mathrm{H}, \mathrm{CH}_{3}\right), 2.12-2.06\left(\mathrm{~m}, 4 \mathrm{H}, 2 \times \mathrm{CH}_{2}\right), 1.68\left(\mathrm{~s}, 3 \mathrm{H}, \mathrm{CH}_{3}\right), 1.61\left(\mathrm{~s}, 3 \mathrm{H}, \mathrm{CH}_{3}\right), 1.16(\mathrm{~d}, J=6.6 \mathrm{~Hz}$, $6 \mathrm{H}, 2 \times \mathrm{CH}_{3}$ ); HR-MS (ESI) $m / z: \mathrm{C}_{12} \mathrm{H}_{21} \mathrm{NO}[\mathrm{M}+\mathrm{H}]^{+}$, Calcd. 196.1696; Found 196.1694. The spectral data were identical with those in the reference [29].

(Z)-N-Phenyl-3,7-dimethyl-2,6-octadienamide (5A), brown oil, yield 70\%. ${ }^{1} \mathrm{H}-\mathrm{NMR}\left(300 \mathrm{MHz}, \mathrm{CDCl}_{3}\right) \delta$ : $7.52(\mathrm{~d}, J=7.8 \mathrm{~Hz}, 2 \mathrm{H}, \mathrm{ArH}), 7.33-7.28(\mathrm{~m}, 2 \mathrm{H}, \mathrm{ArH}), 7.16(\mathrm{~s}, 1 \mathrm{H}, \mathrm{NH}), 7.11-7.06(\mathrm{~m}, 1 \mathrm{H}, \mathrm{ArH}), 5.70$ $(\mathrm{s}, 1 \mathrm{H},=\mathrm{CH}), 5.22-5.16(\mathrm{~m}, 1 \mathrm{H},=\mathrm{CH}), 2.70\left(\mathrm{t}, J=7.8 \mathrm{~Hz}, 2 \mathrm{H}, \mathrm{CH}_{2}\right), 2.26-2.18\left(\mathrm{~m}, 2 \mathrm{H}, \mathrm{CH}_{2}\right), 1.89(\mathrm{~d}$, $\left.J=1.2 \mathrm{~Hz}, 3 \mathrm{H}, \mathrm{CH}_{3}\right), 1.68\left(\mathrm{~s}, 3 \mathrm{H}, \mathrm{CH}_{3}\right), 1.63\left(\mathrm{~s}, 3 \mathrm{H}, \mathrm{CH}_{3}\right)$; HR-MS (ESI) $m / z: \mathrm{C}_{16} \mathrm{H}_{21} \mathrm{NO}[\mathrm{M}+\mathrm{H}]^{+}$, Calcd. 244.1696; Found 244.1691. The spectral data were identical with those in the reference [29].

(Z)-N-(2,4-Dichlorophenyl)-3,7-dimethyl-2,6-octadienamide (5B), yellow oil, yield 65\%. ${ }^{1} \mathrm{H}-\mathrm{NMR}(300 \mathrm{MHz}$, $\left.\mathrm{CDCl}_{3}\right) \delta: 8.43(\mathrm{~d}, J=8.7 \mathrm{~Hz}, 1 \mathrm{H}, \mathrm{ArH}), 7.47(\mathrm{~s}, 1 \mathrm{H}, \mathrm{NH}), 7.37(\mathrm{~d}, J=2.4 \mathrm{~Hz}, 1 \mathrm{H}, \mathrm{ArH}), 7.22(\mathrm{dd}, J=8.7$, $2.4 \mathrm{~Hz}, 1 \mathrm{H}, \mathrm{ArH}), 5.73(\mathrm{~s}, 1 \mathrm{H},=\mathrm{CH}), 5.18-5.14(\mathrm{~m}, 1 \mathrm{H},=\mathrm{CH}), 2.71\left(\mathrm{t}, J=7.5 \mathrm{~Hz}, 2 \mathrm{H}, \mathrm{CH}_{2}\right), 2.26-2.17$ $\left(\mathrm{m}, 2 \mathrm{H}, \mathrm{CH}_{2}\right), 1.93\left(\mathrm{~d}, J=1.2 \mathrm{~Hz}, 3 \mathrm{H}, \mathrm{CH}_{3}\right), 1.67\left(\mathrm{~s}, 3 \mathrm{H}, \mathrm{CH}_{3}\right), 1.63\left(\mathrm{~s}, 3 \mathrm{H}, \mathrm{CH}_{3}\right) ; \mathrm{HR}-\mathrm{MS}(\mathrm{ESI}) \mathrm{m} / z$ : $\mathrm{C}_{16} \mathrm{H}_{19} \mathrm{Cl}_{2} \mathrm{NO}[\mathrm{M}+\mathrm{H}]^{+}$, Calcd. 312.0916; Found 312.0915.

(Z)-N-(2-Chlorophenyl)-3,7-dimethyl-2,6-octadienamide (5C), brown oil, yield 67\%. ${ }^{1} \mathrm{H}-\mathrm{NMR}(300 \mathrm{MHz}$, $\left.\mathrm{CDCl}_{3}\right) \delta: 8.44(\mathrm{~d}, J=8.0 \mathrm{~Hz}, 1 \mathrm{H}, \mathrm{ArH}), 7.54(\mathrm{~s}, 1 \mathrm{H}, \mathrm{NH}), 7.34(\mathrm{dd}, J=8.0,1.5 \mathrm{~Hz}, 1 \mathrm{H}, \mathrm{ArH}), 7.28-7.23$ $(\mathrm{m}, 1 \mathrm{H}, \mathrm{ArH}), 7.04-6.98(\mathrm{~m}, 1 \mathrm{H}, \mathrm{ArH}), 5.75(\mathrm{~s}, 1 \mathrm{H},=\mathrm{CH}), 5.19-5.14(\mathrm{~m}, 1 \mathrm{H}, \mathrm{CH}), 2.72(\mathrm{t}, J=7.5 \mathrm{~Hz}$, $\left.2 \mathrm{H}, \mathrm{CH}_{2}\right), 2.24-2.18\left(\mathrm{~m}, 2 \mathrm{H}, \mathrm{CH}_{2}\right), 1.93\left(\mathrm{~d}, \mathrm{~J}=1.2 \mathrm{~Hz}, 3 \mathrm{H}, \mathrm{CH}_{3}\right), 1.68\left(\mathrm{~s}, 3 \mathrm{H}, \mathrm{CH}_{3}\right), 1.63\left(\mathrm{~s}, 3 \mathrm{H}, \mathrm{CH}_{3}\right)$; HR-MS (ESI) $m / z: \mathrm{C}_{16} \mathrm{H}_{20} \mathrm{ClNO}[\mathrm{M}+\mathrm{H}]^{+}$, Calcd. 278.1306; Found 278.1304.

(Z)-N-(2-Fluorophenyl)-3,7-dimethyl-2,6-octadienamide (5D), brown oil, 15\%. ${ }^{1} \mathrm{H}-\mathrm{NMR}(300 \mathrm{MHz}$, $\left.\mathrm{CDCl}_{3}\right) \delta: 8.43-8.36(\mathrm{~m}, 1 \mathrm{H}, \mathrm{ArH}), 7.26(\mathrm{~s}, 1 \mathrm{H}, \mathrm{NH}), 7.15-6.99(\mathrm{~m}, 3 \mathrm{H}, \mathrm{ArH}), 5.73(\mathrm{~s}, 1 \mathrm{H},=\mathrm{CH})$, 5.19-5.10 (m, 1H, =CH), $2.72\left(\mathrm{t}, J=7.5 \mathrm{~Hz}, 2 \mathrm{H}, \mathrm{CH}_{2}\right), 2.24-2.17\left(\mathrm{~m}, 2 \mathrm{H}, \mathrm{CH}_{2}\right), 1.92(\mathrm{~d}, J=1.2 \mathrm{~Hz}$, $\left.3 \mathrm{H}, \mathrm{CH}_{3}\right), 1.70\left(\mathrm{~s}, 3 \mathrm{H}, \mathrm{CH}_{3}\right), 1.63\left(\mathrm{~s}, 3 \mathrm{H}, \mathrm{CH}_{3}\right) ; \mathrm{HR}-\mathrm{MS}(\mathrm{ESI}) \mathrm{m} / z: \mathrm{C}_{16} \mathrm{H}_{20} \mathrm{FNO}[\mathrm{M}+\mathrm{H}]^{+}$, Calcd. 262.1601; Found 262.1597.

(Z)-3,7-Dimethyl-N-(4-(trifluoromethyl)phenyl)octa-2,6-dienamide (5E), brown oil, yield 16\%. ${ }^{1} \mathrm{H}-\mathrm{NMR}$ $\left(300 \mathrm{MHz}, \mathrm{CDCl}_{3}\right)$ \&: $7.66(\mathrm{~d}, J=8.7 \mathrm{~Hz}, 2 \mathrm{H}, \mathrm{ArH}), 7.55(\mathrm{~d}, J=8.7 \mathrm{~Hz}, 2 \mathrm{H}, \mathrm{ArH}), 7.29(\mathrm{~s}, 1 \mathrm{H}, \mathrm{NH}), 5.71$ $(\mathrm{s}, 1 \mathrm{H},=\mathrm{CH}), 5.19-5.11(\mathrm{~m}, 1 \mathrm{H},=\mathrm{CH}), 2.71\left(\mathrm{t}, J=7.5 \mathrm{~Hz}, 2 \mathrm{H}, \mathrm{CH}_{2}\right), 2.26-2.21\left(\mathrm{~m}, 2 \mathrm{H}, \mathrm{CH}_{2}\right), 1.92(\mathrm{~d}$, $\left.J=1.2 \mathrm{~Hz}, 3 \mathrm{H}, \mathrm{CH}_{3}\right), 1.69\left(\mathrm{~s}, 3 \mathrm{H}, \mathrm{CH}_{3}\right), 1.63\left(\mathrm{~s}, 3 \mathrm{H}, \mathrm{CH}_{3}\right) ; \mathrm{HR}-\mathrm{MS}(\mathrm{ESI}) \mathrm{m} / z: \mathrm{C}_{17} \mathrm{H}_{20} \mathrm{~F}_{3} \mathrm{NO}[\mathrm{M}+\mathrm{H}]^{+}$, Calcd. 312.1569; Found 312.1565.

(Z)-N-(4-Methylphenyl)-3,7-dimethyl-2,6-octadienamide (5F), brown oil, yield 60\%. ${ }^{1} \mathrm{H}-\mathrm{NMR}(300 \mathrm{MHz}$, $\left.\mathrm{CDCl}_{3}\right) \delta: 7.40(\mathrm{~d}, J=7.8 \mathrm{~Hz}, 2 \mathrm{H}, \mathrm{ArH}), 7.11(\mathrm{~d}, J=7.8 \mathrm{~Hz}, 2 \mathrm{H}, \mathrm{ArH}), 7.07(\mathrm{~s}, 1 \mathrm{H}, \mathrm{NH}), 5.68(\mathrm{~s}, 1 \mathrm{H}$, $=\mathrm{CH}), 5.20-5.16(\mathrm{~m}, 1 \mathrm{H},=\mathrm{CH}), 2.69\left(\mathrm{t}, J=7.5 \mathrm{~Hz}, 2 \mathrm{H}, \mathrm{CH}_{2}\right), 2.31\left(\mathrm{~s}, 3 \mathrm{H}, \mathrm{CH}_{3}\right), 2.25-2.16\left(\mathrm{~m}, 2 \mathrm{H}, \mathrm{CH}_{2}\right)$, $1.89\left(\mathrm{~d}, J=1.2 \mathrm{~Hz}, 3 \mathrm{H}, \mathrm{CH}_{3}\right), 1.68\left(\mathrm{~s}, 3 \mathrm{H}, \mathrm{CH}_{3}\right), 1.62\left(\mathrm{~s}, 3 \mathrm{H}, \mathrm{CH}_{3}\right)$; HR-MS (ESI) $m / z: \mathrm{C}_{17} \mathrm{H}_{23} \mathrm{NO}$ $[\mathrm{M}+\mathrm{H}]^{+}$, Calcd. 258.1852; Found 258.1850.

(Z)-N-Phenyl-N,3,7-trimethyl-2,6-octadienamide (5G), brown oil, yield $67 \% .{ }^{1} \mathrm{H}-\mathrm{NMR}\left(300 \mathrm{MHz}, \mathrm{CDCl}_{3}\right)$

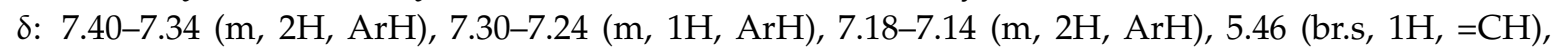
$5.22-5.16(\mathrm{~m}, 1 \mathrm{H},=\mathrm{CH}), 3.31\left(\mathrm{~s}, 3 \mathrm{H}, \mathrm{NCH}_{3}\right), 2.61\left(\mathrm{t}, J=7.5 \mathrm{~Hz}, 2 \mathrm{H}, \mathrm{CH}_{2}\right), 2.22-2.14\left(\mathrm{~m}, 2 \mathrm{H}, \mathrm{CH}_{2}\right), 1.70$ 
$\left(\mathrm{d}, J=1.2 \mathrm{~Hz}, 3 \mathrm{H}, \mathrm{CH}_{3}\right), 1.67\left(\mathrm{~s}, 3 \mathrm{H}, \mathrm{CH}_{3}\right), 1.64\left(\mathrm{~s}, 3 \mathrm{H}, \mathrm{CH}_{3}\right)$; HR-MS (ESI) $m / z: \mathrm{C}_{17} \mathrm{H}_{23} \mathrm{NO}[\mathrm{M}+\mathrm{H}]^{+}$, Calcd. 258.1852; Found 258.1849. The spectral data were identical with those in the reference [31].

(Z)-N-Benzyl-3,7-dimethyl-2,6-octadienamide (5H), yellow oil, yield 57\%. ${ }^{1} \mathrm{H}-\mathrm{NMR}\left(300 \mathrm{MHz}, \mathrm{CDCl}_{3}\right) \delta$ : 7.31-7.26 (m, 5H, ArH), 5.66 (br.s, $1 \mathrm{H}, \mathrm{NH}), 5.58(\mathrm{~s}, 1 \mathrm{H},=\mathrm{CH}), 5.18-5.12(\mathrm{~m}, 1 \mathrm{H},=\mathrm{CH}), 4.47(\mathrm{~d}, J=6.0 \mathrm{~Hz}$, $\left.2 \mathrm{H}, \mathrm{ArCH}_{2}\right), 2.64\left(\mathrm{t}, J=7.8 \mathrm{~Hz}, 2 \mathrm{H}, \mathrm{CH}_{2}\right), 2.22-2.18\left(\mathrm{~m}, 2 \mathrm{H}, \mathrm{CH}_{2}\right), 1.84\left(\mathrm{~d}, J=1.2 \mathrm{~Hz}, 3 \mathrm{H}, \mathrm{CH}_{3}\right), 1.64(\mathrm{~s}$, $\left.3 \mathrm{H}, \mathrm{CH}_{3}\right), 1.60\left(\mathrm{~s}, 3 \mathrm{H}, \mathrm{CH}_{3}\right.$ ); HR-MS (ESI) $m / z: \mathrm{C}_{17} \mathrm{H}_{23} \mathrm{NO}[\mathrm{M}+\mathrm{H}]^{+}$, Calcd. 258.1852; Found 258.1848. The spectral data were identical with those in the reference [30].

(Z)-N-(4-Fluorobenzyl)-3,7-dimethyl-2,6-octadienamide (5I), brown oil, yield 72\%. ${ }^{1} \mathrm{H}-\mathrm{NMR}(300 \mathrm{MHz}$, $\left.\mathrm{CDCl}_{3}\right)$ 8: 7.29-7.23 (m, 2H, ArH), 7.03-6.97 (m, 2H, ArH), 5.71 (br.s, 1H, NH), $5.58(\mathrm{~s}, 1 \mathrm{H},=\mathrm{CH})$, 5.17-5.11 (m, 1H, =CH), $4.43\left(\mathrm{~d}, J=5.8 \mathrm{~Hz}, 2 \mathrm{H}, \mathrm{ArCH}_{2}\right), 2.64\left(\mathrm{t}, J=7.2 \mathrm{~Hz}, 2 \mathrm{H}, \mathrm{CH}_{2}\right), 2.21-2.16(\mathrm{~m}, 2 \mathrm{H}$, $\left.\mathrm{CH}_{2}\right), 1.84\left(\mathrm{~d}, J=1.2 \mathrm{~Hz}, 3 \mathrm{H}, \mathrm{CH}_{3}\right), 1.65\left(\mathrm{~s}, 3 \mathrm{H}, \mathrm{CH}_{3}\right), 1.60\left(\mathrm{~s}, 3 \mathrm{H}, \mathrm{CH}_{3}\right) ; \mathrm{HR}-\mathrm{MS}(\mathrm{ESI}) \mathrm{m} / z: \mathrm{C}_{17} \mathrm{H}_{22} \mathrm{FNO}$ $[\mathrm{M}+\mathrm{H}]^{+}$: Calcd. 276.1758; Found 276.1754.

(Z)-N-(4-Methoxybenzyl)-3,7-dimethyl-2,6-octadienamide (5J), yellow oil, 27\%. ${ }^{1} \mathrm{H}-\mathrm{NMR}(300 \mathrm{MHz}$, $\left.\mathrm{CDCl}_{3}\right) \delta: 7.20(\mathrm{~d}, J=8.4 \mathrm{~Hz}, 2 \mathrm{H}, \mathrm{ArH}), 6.84(\mathrm{~d}, J=8.4 \mathrm{~Hz}, 2 \mathrm{H}, \mathrm{ArH}), 5.71(\mathrm{~s}, 1 \mathrm{H}, \mathrm{NH}), 5.56(\mathrm{~s}, 1 \mathrm{H}$, $=\mathrm{CH}), 5.17-5.11(\mathrm{~m}, 1 \mathrm{H},=\mathrm{CH}), 4.38\left(\mathrm{~d}, J=6.0 \mathrm{~Hz}, 2 \mathrm{H}, \mathrm{ArCH}_{2}\right), 3.79\left(\mathrm{~s}, 3 \mathrm{H}, \mathrm{OCH}_{3}\right), 2.64(\mathrm{t}, J=7.5 \mathrm{~Hz}$, $\left.2 \mathrm{H}, \mathrm{CH}_{2}\right), 2.21-2.13\left(\mathrm{~m}, 2 \mathrm{H}, \mathrm{CH}_{2}\right), 1.83\left(\mathrm{~d}, J=1.2 \mathrm{~Hz}, 3 \mathrm{H}, \mathrm{CH}_{3}\right), 1.64\left(\mathrm{~s}, 3 \mathrm{H}, \mathrm{CH}_{3}\right), 1.60\left(\mathrm{~s}, 3 \mathrm{H}, \mathrm{CH}_{3}\right)$; HR-MS (ESI) $m / z: \mathrm{C}_{18} \mathrm{H}_{25} \mathrm{FNO}_{2}[\mathrm{M}+\mathrm{H}]^{+}$: Calcd. 288.1958; Found 288.1955.

(Z)-N-(2-Chlorobenzyl)-3,7-dimethyl-2,6-octadienamide (5K), yellow oil, 40\%. ${ }^{1} \mathrm{H}-\mathrm{NMR}(300 \mathrm{MHz}$, $\left.\mathrm{CDCl}_{3}\right)$ 8: 7.40-7.33 (m, 2H, ArH), 7.24-7.19 (m, 2H, ArH), $5.90(\mathrm{~s}, 1 \mathrm{H}, \mathrm{NH}), 5.59(\mathrm{~s}, 1 \mathrm{H},=\mathrm{CH})$, 5.15-5.10 (m, 1H, =CH), $4.54\left(\mathrm{~d}, J=6.0 \mathrm{~Hz}, 2 \mathrm{H}, \mathrm{CH}_{2}\right), 2.63\left(\mathrm{t}, J=7.5 \mathrm{~Hz}, 2 \mathrm{H}, \mathrm{CH}_{2}\right), 2.20-2.11(\mathrm{~m}$, $\left.2 \mathrm{H}, \mathrm{CH}_{2}\right), 1.83\left(\mathrm{~d}, J=1.2 \mathrm{~Hz}, 3 \mathrm{H}, \mathrm{CH}_{3}\right), 1.64\left(\mathrm{~s}, 3 \mathrm{H}, \mathrm{CH}_{3}\right), 1.59\left(\mathrm{~s}, 3 \mathrm{H}, \mathrm{CH}_{3}\right) ; \mathrm{HR}-\mathrm{MS}(\mathrm{ESI}) \mathrm{m} / z$ : $\mathrm{C}_{17} \mathrm{H}_{22} \mathrm{ClNO}[\mathrm{M}+\mathrm{H}]^{+}$: Calcd. 292.1462; Found 292.1461.

(Z)-3,7-Dimethyl-1-morpholino-2,6-octadien-1-one (5L), yellow oil, yield 60\%. ${ }^{1} \mathrm{H}-\mathrm{NMR}(300 \mathrm{MHz}$, $\left.\mathrm{CDCl}_{3}\right) \delta: 5.74(\mathrm{~s}, 1 \mathrm{H},=\mathrm{CH}), 5.13-5.07(\mathrm{~m}, 1 \mathrm{H},=\mathrm{CH}), 3.66\left(\mathrm{br}, 6 \mathrm{H}, 3 \times \mathrm{CH}_{2}\right), 3.50\left(\mathrm{br}, 2 \mathrm{H}, \mathrm{CH}_{2}\right)$, $2.33\left(\mathrm{t}, J=7.5 \mathrm{~Hz}, 2 \mathrm{H}, \mathrm{CH}_{2}\right), 2.18-2.10\left(\mathrm{~m}, 2 \mathrm{H}, \mathrm{CH}_{2}\right), 1.83\left(\mathrm{~d}, J=1.2 \mathrm{~Hz}, 3 \mathrm{H}, \mathrm{CH}_{3}\right), 1.68\left(\mathrm{~s}, 3 \mathrm{H}, \mathrm{CH}_{3}\right)$, $1.61\left(\mathrm{~s}, 3 \mathrm{H}, \mathrm{CH}_{3}\right)$; HR-MS (ESI) $m / z: \mathrm{C}_{14} \mathrm{H}_{23} \mathrm{NO}_{2}[\mathrm{M}+\mathrm{H}]^{+}$, Calcd. 238.1802; Found 238.1800.

(Z)-3,7-Dimethyl-1-(pyrrolidin-1-yl)-2,6-octadien-1-one (5M), pale yellow oil, yield 65\%. ${ }^{1} \mathrm{H}-\mathrm{NMR}$ $\left(300 \mathrm{MHz}, \mathrm{CDCl}_{3}\right)$ 8: $5.77(\mathrm{~s}, 1 \mathrm{H},=\mathrm{CH}), 5.18-5.11(\mathrm{~m}, 1 \mathrm{H},=\mathrm{CH}), 3.49\left(\mathrm{t}, J=6.9 \mathrm{~Hz}, 2 \mathrm{H}, \mathrm{NCH}_{2}\right)$, $3.42\left(\mathrm{t}, J=6.9 \mathrm{~Hz}, 2 \mathrm{H}, \mathrm{NCH}_{2}\right), 2.53\left(\mathrm{t}, J=7.5 \mathrm{~Hz}, 2 \mathrm{H}, \mathrm{CH}_{2}\right), 2.20-2.15\left(\mathrm{~m}, 2 \mathrm{H}, \mathrm{CH}_{2}\right), 1.95-1.82(\mathrm{~m}, 7 \mathrm{H}$, $\left.2 \times \mathrm{CH}_{2}+\mathrm{CH}_{3}\right), 1.67\left(\mathrm{~s}, 3 \mathrm{H}, \mathrm{CH}_{3}\right), 1.61\left(\mathrm{~s}, 3 \mathrm{H}, \mathrm{CH}_{3}\right) ; \mathrm{HR}-\mathrm{MS}(\mathrm{ESI}) \mathrm{m} / z: \mathrm{C}_{14} \mathrm{H}_{23} \mathrm{NO}[\mathrm{M}+\mathrm{H}]^{+}, \mathrm{Calcd}$. 222.1852; Found 222.1850. The spectral data were identical with those in the reference [28].

(Z)-N-Isopropyl-3,7-dimethyl-2,6-octadienamide (5N), yellow oil, yield $62 \% .{ }^{1} \mathrm{H}-\mathrm{NMR}\left(300 \mathrm{MHz}, \mathrm{CDCl}_{3}\right)$ $\delta: 5.51(\mathrm{~s}, 1 \mathrm{H},=\mathrm{CH}), 5.27$ (br.s, $1 \mathrm{H}, \mathrm{NH}), 5.19-5.14(\mathrm{~m}, 1 \mathrm{H},=\mathrm{CH}), 4.15-4.08(\mathrm{~m}, 1 \mathrm{H}, \mathrm{NCH}), 2.59(\mathrm{t}$, $\left.J=7.2 \mathrm{~Hz}, 2 \mathrm{H}, \mathrm{CH}_{2}\right), 2.21-2.14\left(\mathrm{~m}, 2 \mathrm{H}, \mathrm{CH}_{2}\right), 1.86\left(\mathrm{~d}, J=1.2 \mathrm{~Hz}, 3 \mathrm{H}, \mathrm{CH}_{3}\right), 1.69\left(\mathrm{~s}, 3 \mathrm{H}, \mathrm{CH}_{3}\right), 1.62(\mathrm{~s}$, $\left.3 \mathrm{H}, \mathrm{CH}_{3}\right), 1.16\left(\mathrm{~d}, \mathrm{~J}=6.6 \mathrm{~Hz}, 6 \mathrm{H}, 2 \times \mathrm{CH}_{3}\right)$; HR-MS (ESI) $m / z: \mathrm{C}_{12} \mathrm{H}_{21} \mathrm{NO}[\mathrm{M}+\mathrm{H}]^{+}$, Calcd. 196.1696; Found 196.1694. The spectral data were identical with those in the reference [28].

\subsubsection{General Procedure for the Synthesis of Compounds 6}

Take compound $\mathbf{6 a}$ as an example: according to the approach in the literature [25,32], compound 5 a $0.24 \mathrm{~g}(1 \mathrm{mmol}), \mathrm{CH}_{3} \mathrm{COOOH}(2 \mathrm{~mL}), \mathrm{Na}_{2} \mathrm{CO}_{3}(0.7 \mathrm{~g})$ and $\mathrm{DCM}(10 \mathrm{~mL})$ were added to a $50 \mathrm{~mL}$ flask and stirred at room temperature for $2-4 \mathrm{~h}$, quenched the reaction with water, and extracted with DCM. The organic layer was washed with brine and dried over anhydrous $\mathrm{Na}_{2} \mathrm{SO}_{4}$. The solvent was removed under reduce pressure, and the residue was purified by column chromatography to give the compounds $6 \mathbf{a}$.

(E)-N-Phenyl-5-(3,3-dimethyloxiran-2-yl)-3-methylpent-2-enamide (6a), yellow solid, yield 80\%. m.p. 82-84 ${ }^{\circ} \mathrm{C},{ }^{1} \mathrm{H}-\mathrm{NMR}\left(300 \mathrm{MHz}, \mathrm{CDCl}_{3}\right) \delta: 7.54(\mathrm{~d}, J=7.8 \mathrm{~Hz}, 2 \mathrm{H}, \mathrm{ArH}), 7.29(\mathrm{t}, J=7.8 \mathrm{~Hz}, 2 \mathrm{H}, \mathrm{ArH})$, $7.21(\mathrm{~s}, 1 \mathrm{H}, \mathrm{NH}), 7.09(\mathrm{t}, J=7.8 \mathrm{~Hz}, 1 \mathrm{H}, \mathrm{ArH}), 5.76(\mathrm{~s}, 1 \mathrm{H},=\mathrm{CH}), 2.74(\mathrm{dd}, J=5.1,7.2 \mathrm{~Hz}, 1 \mathrm{H}, \mathrm{OCH})$, 
2.36-2.25 (m, 2H, $\left.\mathrm{CH}_{2}\right), 2.24\left(\mathrm{~d}, J=1.2 \mathrm{~Hz}, 3 \mathrm{H}, \mathrm{CH}_{3}\right), 1.78-1.66\left(\mathrm{~m}, 2 \mathrm{H}, \mathrm{CH}_{2}\right), 1.33\left(\mathrm{~s}, 3 \mathrm{H}, \mathrm{CH}_{3}\right), 1.29$ (s, 3H, $\mathrm{CH}_{3}$ ); HR-MS (ESI) $m / z: \mathrm{C}_{16} \mathrm{H}_{21} \mathrm{NO}_{2}[\mathrm{M}+\mathrm{H}]^{+}$, Calcd. 260.1645; Found 260.1643.

(E)-N-(2,4-Dichlorophenyl)-5-(3,3-dimethyloxiran-2-yl)-3-methylpent-2-enamide (6b), yellow oil, yield 63\%. ${ }^{1} \mathrm{H}-\mathrm{NMR}\left(300 \mathrm{MHz}, \mathrm{CDCl}_{3}\right) \delta: 8.42(\mathrm{~d}, J=8.7 \mathrm{~Hz}, 1 \mathrm{H}, \mathrm{ArH}), 7.53(\mathrm{~s}, 1 \mathrm{H}, \mathrm{NH}), 7.26(\mathrm{~d}, J=2.4 \mathrm{~Hz}$, $1 \mathrm{H}, \mathrm{ArH}), 7.24(\mathrm{dd}, J=8.7,2.4 \mathrm{~Hz}, 1 \mathrm{H}, \mathrm{ArH}), 5.80(\mathrm{~s}, 1 \mathrm{H},=\mathrm{CH}), 2.74(\mathrm{dd}, J=7.2,5.1 \mathrm{~Hz}, 1 \mathrm{H}, \mathrm{OCH})$, 2.40-2.30 (m, $\left.2 \mathrm{H}, \mathrm{CH}_{2}\right), 2.25\left(\mathrm{~d}, J=1.2 \mathrm{~Hz}, 3 \mathrm{H}, \mathrm{CH}_{3}\right), 1.82-1.66\left(\mathrm{~m}, 2 \mathrm{H}, \mathrm{CH}_{2}\right), 1.33\left(\mathrm{~s}, 3 \mathrm{H}, \mathrm{CH}_{3}\right), 1.30$ (s, $3 \mathrm{H}, \mathrm{CH}_{3}$ ); HR-MS (ESI) $m / z: \mathrm{C}_{16} \mathrm{H}_{19} \mathrm{Cl}_{2} \mathrm{NO}_{2}[\mathrm{M}+\mathrm{H}]^{+}$, Calcd. 328.0866; Found 328.0864.

(E)-N-(2-Chlorophenyl)-5-(3,3-dimethyloxiran-2-yl)-3-methylpent-2-enamide (6c), yellow oil, yield 80\%. ${ }^{1} \mathrm{H}-\mathrm{NMR}\left(300 \mathrm{MHz}, \mathrm{CDCl}_{3}\right) \delta: 8.44(\mathrm{~d}, J=7.8 \mathrm{~Hz}, 1 \mathrm{H}, \mathrm{ArH}), 7.59(\mathrm{~s}, 1 \mathrm{H}, \mathrm{NH}), 7.36(\mathrm{dd}, J=7.8$, $1.8 \mathrm{~Hz}, 1 \mathrm{H}, \mathrm{ArH}), 7.30-7.23(\mathrm{~m}, 1 \mathrm{H}, \mathrm{ArH}), 7.05-6.99(\mathrm{~m}, 1 \mathrm{H}, \mathrm{ArH}), 5.81(\mathrm{~s}, 1 \mathrm{H},=\mathrm{CH}), 2.74(\mathrm{dd}, J=7.2$, $5.4 \mathrm{~Hz}, 1 \mathrm{H}, \mathrm{OCH}), 2.40-2.29\left(\mathrm{~m}, 2 \mathrm{H}, \mathrm{CH}_{2}\right), 2.25\left(\mathrm{~d}, J=1.2 \mathrm{~Hz}, 3 \mathrm{H}, \mathrm{CH}_{3}\right), 1.82-1.67\left(\mathrm{~m}, 2 \mathrm{H}, \mathrm{CH}_{2}\right), 1.33$ (s, 3H, $\left.\mathrm{CH}_{3}\right), 1.30\left(\mathrm{~s}, 3 \mathrm{H}, \mathrm{CH}_{3}\right)$; HR-MS (ESI) $\mathrm{m} / z: \mathrm{C}_{16} \mathrm{H}_{20} \mathrm{ClNO}_{2}[\mathrm{M}+\mathrm{H}]^{+}$, Calcd. 294.1255; Found 294.1257.

(E)-N-(4-Methylphenyl)-5-(3,3-dimethyloxiran-2-yl)-3-methylpent-2-enamide (6d), colorless oil, yield 75\%. ${ }^{1} \mathrm{H}-\mathrm{NMR}\left(300 \mathrm{MHz}, \mathrm{CDCl}_{3}\right)$ \&: $7.42(\mathrm{~d}, J=7.8 \mathrm{~Hz}, 2 \mathrm{H}, \mathrm{ArH}), 7.11(\mathrm{~d}, J=7.8 \mathrm{~Hz}, 3 \mathrm{H}, \mathrm{ArH}+\mathrm{NH}), 5.74$ (s, $1 \mathrm{H},=\mathrm{CH}), 2.74(\mathrm{dd}, J=7.2,5.1 \mathrm{~Hz}, 1 \mathrm{H}, \mathrm{OCH}), 2.35-2.25\left(\mathrm{~m}, 5 \mathrm{H}, \mathrm{CH}_{2}+\mathrm{CH}_{3}\right), 2.23(\mathrm{~d}, J=1.2 \mathrm{~Hz}$, $\left.3 \mathrm{H}, \mathrm{CH}_{3}\right), 1.80-1.64\left(\mathrm{~m}, 2 \mathrm{H}, \mathrm{CH}_{2}\right), 1.32\left(\mathrm{~s}, 3 \mathrm{H}, \mathrm{CH}_{3}\right), 1.29\left(\mathrm{~s}, 3 \mathrm{H}, \mathrm{CH}_{3}\right)$; HR-MS (ESI) $m / z: \mathrm{C}_{17} \mathrm{H}_{23} \mathrm{NO}_{2}$ $[\mathrm{M}+\mathrm{H}]^{+}$, Calcd. 274.1802; Found 274.1798.

(E)-N-Phenyl-5-(3,3-dimethyloxiran-2-yl)-N,3-dimethylpent-2-enamide (6e), yellow oil, yield 96\%. ${ }^{1} \mathrm{H}-\mathrm{NMR}\left(300 \mathrm{MHz}, \mathrm{CDCl}_{3}\right)$ 8: 7.40-7.33 (m, 2H, ArH), 7.31-7.25 (m, 1H, ArH), 7.17-7.13 (m, 2H, ArH), 5.51 (br.s, $1 \mathrm{H},=\mathrm{CH}), 3.32\left(\mathrm{~s}, 3 \mathrm{H}, \mathrm{CH}_{3}\right), 2.55(\mathrm{t}, J=6.3 \mathrm{~Hz}, 1 \mathrm{H}, \mathrm{OCH}), 2.11(\mathrm{~d}, J=1.2 \mathrm{~Hz}, 3 \mathrm{H}$, $\left.\mathrm{CH}_{3}\right), 2.08-2.03\left(\mathrm{~m}, 2 \mathrm{H}, \mathrm{CH}_{2}\right), 1.53-1.45\left(\mathrm{~m}, 2 \mathrm{H}, \mathrm{CH}_{2}\right), 1.25\left(\mathrm{~s}, 3 \mathrm{H}, \mathrm{CH}_{3}\right), 1.18\left(\mathrm{~s}, 3 \mathrm{H}, \mathrm{CH}_{3}\right) ; \mathrm{HR}-\mathrm{MS}$ (ESI) $m / z: \mathrm{C}_{17} \mathrm{H}_{23} \mathrm{NO}_{2}[\mathrm{M}+\mathrm{H}]^{+}$, Calcd. 274.1802; Found 274.1792.

(E)-N-Benzyl-5-(3,3-dimethyloxiran-2-yl)-3-methylpent-2-enamide (6f), yellow oil, yield 80\%. ${ }^{1} \mathrm{H}-\mathrm{NMR}$ $\left(300 \mathrm{MHz}, \mathrm{CDCl}_{3}\right)$ 8: 7.36-7.25 (m, 5H, ArH), 5.68 (br.s, $\left.1 \mathrm{H}, \mathrm{NH}\right), 5.62(\mathrm{~s}, 1 \mathrm{H},=\mathrm{CH}), 4.47(\mathrm{~d}, J=6.0 \mathrm{~Hz}$, $\left.2 \mathrm{H}, \mathrm{ArCH}_{2}\right), 2.71(\mathrm{dd}, J=7.2,5.4 \mathrm{~Hz}, 1 \mathrm{H}, \mathrm{OCH}), 2.30-2.22\left(\mathrm{~m}, 2 \mathrm{H}, \mathrm{CH}_{2}\right), 2.20\left(\mathrm{~d}, J=1.2 \mathrm{~Hz}, 3 \mathrm{H}, \mathrm{CH}_{3}\right)$, $1.75-1.62\left(\mathrm{~m}, 2 \mathrm{H}, \mathrm{CH}_{2}\right), 1.32\left(\mathrm{~s}, 3 \mathrm{H}, \mathrm{CH}_{3}\right), 1.26\left(\mathrm{~s}, 3 \mathrm{H}, \mathrm{CH}_{3}\right)$; HR-MS (ESI) $m / z: \mathrm{C}_{17} \mathrm{H}_{23} \mathrm{NO}_{2}[\mathrm{M}+\mathrm{H}]^{+}$, Calcd. 274.1802; Found 274.1799.

(E)-N-(4-Fluorobenzyl)-5-(3,3-dimethyloxiran-2-yl)-3-methylpent-2-enamide (6g), white solid, yield 84\%. m.p. 50-52 ${ }^{\circ} \mathrm{C},{ }^{1} \mathrm{H}-\mathrm{NMR}\left(300 \mathrm{MHz}, \mathrm{CDCl}_{3}\right)$ 8: 7.29-7.23 (m, 2H, ArH), 7.04-6.97 (m, 2H, ArH), 5.76 (br.s, $1 \mathrm{H}, \mathrm{NH}), 5.62(\mathrm{~s}, 1 \mathrm{H},=\mathrm{CH}), 4.43\left(\mathrm{~d}, J=5.8 \mathrm{~Hz}, 2 \mathrm{H}, \mathrm{ArCH}_{2}\right), 2.71(\mathrm{dd}, J=7.2,5.1 \mathrm{~Hz}, 1 \mathrm{H}, \mathrm{OCH})$, 2.30-2.19 (m, 5H, $\left.\mathrm{CH}_{2}+\mathrm{CH}_{3}\right), 1.76-1.62\left(\mathrm{~m}, 2 \mathrm{H}, \mathrm{CH}_{2}\right), 1.30\left(\mathrm{~s}, 3 \mathrm{H}, \mathrm{CH}_{3}\right), 1.26\left(\mathrm{~s}, 3 \mathrm{H}, \mathrm{CH}_{3}\right) ; \mathrm{HR}-\mathrm{MS}$ (ESI) $m / z: \mathrm{C}_{17} \mathrm{H}_{22} \mathrm{FNO}_{2}[\mathrm{M}+\mathrm{H}]^{+}$, Calcd. 292.1707; Found 292.1706.

(E)-5-(3,3-Dimethyloxiran-2-yl)-3-methyl-1-morpholinopent-2-en-1-one (6h), pale yellow oil, yield 71\%. ${ }^{1} \mathrm{H}-\mathrm{NMR}\left(300 \mathrm{MHz}, \mathrm{CDCl}_{3}\right)$ ): $5.81(\mathrm{~s}, 1 \mathrm{H},=\mathrm{CH}), 3.67\left(\mathrm{br}, 6 \mathrm{H}, 3 \times \mathrm{CH}_{2}\right), 3.51\left(\mathrm{br}, 2 \mathrm{H}, \mathrm{CH}_{2}\right), 2.71$ $(\mathrm{dd}, J=7.2,5.1 \mathrm{~Hz}, 1 \mathrm{H}, \mathrm{OCH}), 2.32-2.23\left(\mathrm{~m}, 2 \mathrm{H}, \mathrm{CH}_{2}\right), 1.92\left(\mathrm{~d}, J=1.2 \mathrm{~Hz}, 3 \mathrm{H}, \mathrm{CH}_{3}\right), 1.81-1.63(\mathrm{~m}, 2 \mathrm{H}$, $\left.\mathrm{CH}_{2}\right), 1.31\left(\mathrm{~s}, 3 \mathrm{H}, \mathrm{CH}_{3}\right), 1.25$ (s, 3H, $\left.\mathrm{CH}_{3}\right)$; HR-MS (ESI) $m / z: \mathrm{C}_{14} \mathrm{H}_{23} \mathrm{NO}_{3}[\mathrm{M}+\mathrm{H}]^{+}$, Calcd. 254.1751; Found 254.1747.

(E)-5-(3,3-Dimethyloxiran-2-yl)-3-methyl-1-(pyrrolidin-1-yl)pent-2-en-1-one (6i), yellow oil, yield 70\%. ${ }^{1} \mathrm{H}-\mathrm{NMR}\left(300 \mathrm{MHz}, \mathrm{CDCl}_{3}\right)$ 8: $5.84(\mathrm{~s}, 1 \mathrm{H},=\mathrm{CH}), 3.50\left(\mathrm{t}, J=6.6 \mathrm{~Hz}, 2 \mathrm{H}, \mathrm{NCH}_{2}\right), 3.43(\mathrm{t}, J=6.6 \mathrm{~Hz}$, $\left.2 \mathrm{H}, \mathrm{NCH}_{2}\right), 2.72(\mathrm{dd}, J=6.9,5.7 \mathrm{~Hz}, 1 \mathrm{H}, \mathrm{OCH}), 2.32-2.22\left(\mathrm{~m}, 2 \mathrm{H}, \mathrm{CH}_{2}\right), 2.09(\mathrm{~d}, J=1.2 \mathrm{~Hz}$, $\left.3 \mathrm{H}, \mathrm{CH}_{3}\right), 1.96-1.65\left(\mathrm{~m}, 6 \mathrm{H}, 3 \times \mathrm{CH}_{2}\right), 1.31\left(\mathrm{~s}, 3 \mathrm{H}, \mathrm{CH}_{3}\right), 1.28\left(\mathrm{~s}, 3 \mathrm{H}, \mathrm{CH}_{3}\right) ; \mathrm{HR}-\mathrm{MS}(\mathrm{ESI}) \mathrm{m} / z$ : $\mathrm{C}_{14} \mathrm{H}_{23} \mathrm{NO}_{2}[\mathrm{M}+\mathrm{H}]^{+}$, Calcd. 238.1802; Found 238.1799.

(E)-N-Isopropyl-5-(3,3-dimethyloxiran-2-yl)-3-methylpent-2-enamide (6j), yellow oil, yield 79\%. ${ }^{1} \mathrm{H}-\mathrm{NMR}$ $\left(300 \mathrm{MHz}, \mathrm{CDCl}_{3}\right) \delta: 5.55(\mathrm{~s}, 1 \mathrm{H},=\mathrm{CH}), 5.24$ (br.s, 1H, NH), 4.16-4.08 (m, 1H, NCH), $2.72(\mathrm{dd}, J=7.0$, $5.4 \mathrm{~Hz}, 1 \mathrm{H}, \mathrm{OCH}), 2.29-2.14\left(\mathrm{~m}, 5 \mathrm{H}, \mathrm{CH}_{2}+\mathrm{CH}_{3}\right), 1.76-1.62\left(\mathrm{~m}, 2 \mathrm{H}, \mathrm{CH}_{2}\right), 1.31\left(\mathrm{~s}, 3 \mathrm{H}, \mathrm{CH}_{3}\right), 1.27(\mathrm{~s}$, 
$\left.3 \mathrm{H}, \mathrm{CH}_{3}\right), 1.16\left(\mathrm{~d}, J=6.5 \mathrm{~Hz}, 6 \mathrm{H}, 2 \times \mathrm{CH}_{3}\right)$; HR-MS (ESI) $m / z: \mathrm{C}_{13} \mathrm{H}_{24} \mathrm{NO}_{2}[\mathrm{M}+\mathrm{H}]^{+}$, Calcd. 226.1807; Found 226.1799.

(Z)-N-Phenyl-5-(3,3-dimethyloxiran-2-yl)-3-methylpent-2-enamide (6A), brown oil, yield 80\%. ${ }^{1} \mathrm{H}-\mathrm{NMR}$ $\left(300 \mathrm{MHz}, \mathrm{CDCl}_{3}\right) \delta: 7.62(\mathrm{~s}, 1 \mathrm{H}, \mathrm{NH}), 7.54(\mathrm{~d}, J=7.5 \mathrm{~Hz}, 2 \mathrm{H}, \mathrm{ArH}), 7.33-7.26(\mathrm{~m}, 2 \mathrm{H}, \mathrm{ArH}), 7.10-7.04$ $(\mathrm{m}, 1 \mathrm{H}, \mathrm{ArH}), 5.76(\mathrm{~s}, 1 \mathrm{H},=\mathrm{CH}), 2.95-2.60\left(\mathrm{~m}, 3 \mathrm{H}, \mathrm{OCH}+\mathrm{CH}_{2}\right), 1.89\left(\mathrm{~d}, J=1.2 \mathrm{~Hz}, 3 \mathrm{H}, \mathrm{CH}_{3}\right), 1.82-1.72$ $\left(\mathrm{m}, 2 \mathrm{H}, \mathrm{CH}_{2}\right), 1.30\left(\mathrm{~s}, 3 \mathrm{H}, \mathrm{CH}_{3}\right), 1.29\left(\mathrm{~s}, 3 \mathrm{H}, \mathrm{CH}_{3}\right) ; \mathrm{HR}-\mathrm{MS}(\mathrm{ESI}) \mathrm{m} / z: \mathrm{C}_{16} \mathrm{H}_{21} \mathrm{NO}_{2}[\mathrm{M}+\mathrm{H}]^{+}, \mathrm{Calcd}$. 260.1645; Found 260.1641.

(Z)-N-(2,4-Dichlorophenyl)-5-(3,3-dimethyloxiran-2-yl)-3-methylpent-2-enamide (6B), pale yellow oil, yield 68\%. ${ }^{1} \mathrm{H}-\mathrm{NMR}\left(300 \mathrm{MHz}, \mathrm{CDCl}_{3}\right) \delta: 8.37(\mathrm{~d}, J=8.7 \mathrm{~Hz}, 1 \mathrm{H}, \mathrm{ArH}), 7.57(\mathrm{~s}, 1 \mathrm{H}, \mathrm{NH}), 7.35(\mathrm{~d}$, $J=2.1 \mathrm{~Hz}, 1 \mathrm{H}, \mathrm{ArH}), 7.21(\mathrm{dd}, J=8.7,2.1 \mathrm{~Hz}, 1 \mathrm{H}, \mathrm{ArH}), 5.79(\mathrm{~s}, 1 \mathrm{H},=\mathrm{CH}), 2.98-2.73(\mathrm{~m}, 3 \mathrm{H}$, $\left.\mathrm{OCH}+\mathrm{CH}_{2}\right), 1.93\left(\mathrm{~d}, J=1.2 \mathrm{~Hz}, 3 \mathrm{H}, \mathrm{CH}_{3}\right), 1.82-1.74\left(\mathrm{~m}, 2 \mathrm{H}, \mathrm{CH}_{2}\right), 1.30\left(\mathrm{~s}, 3 \mathrm{H}, \mathrm{CH}_{3}\right), 1.29(\mathrm{~s}, 3 \mathrm{H}$, $\mathrm{CH}_{3}$ ); HR-MS (ESI) $m / z: \mathrm{C}_{16} \mathrm{H}_{19} \mathrm{Cl}_{2} \mathrm{NO}_{2}[\mathrm{M}+\mathrm{H}]^{+}$, Calcd. 328.0866; Found 328.0865.

(Z)-N-(2-Chlorophenyl)-5-(3,3-dimethyloxiran-2-yl)-3-methylpent-2-enamide (6C), yellow oil, yield 76\%. ${ }^{1} \mathrm{H}-\mathrm{NMR}\left(300 \mathrm{MHz}, \mathrm{CDCl}_{3}\right) \delta: 8.42(\mathrm{~d}, J=8.4 \mathrm{~Hz}, 1 \mathrm{H}, \mathrm{ArH}), 7.57(\mathrm{~s}, 1 \mathrm{H}, \mathrm{NH}), 7.36(\mathrm{dd}, J=7.8$, $1.5 \mathrm{~Hz}, 1 \mathrm{H}, \mathrm{ArH}), 7.30-7.24(\mathrm{~m}, 1 \mathrm{H}, \mathrm{ArH}), 7.06-6.99(\mathrm{~m}, 1 \mathrm{H}, \mathrm{ArH}), 5.80(\mathrm{~s}, 1 \mathrm{H},=\mathrm{CH}), 2.98-2.74(\mathrm{~m}$, $\left.3 \mathrm{H}, \mathrm{OCH}+\mathrm{CH}_{2}\right), 1.96\left(\mathrm{~d}, J=1.2 \mathrm{~Hz}, 3 \mathrm{H}, \mathrm{CH}_{3}\right), 1.83-1.75\left(\mathrm{~m}, 2 \mathrm{H}, \mathrm{CH}_{2}\right), 1.30\left(\mathrm{~s}, 6 \mathrm{H}, 2 \times \mathrm{CH}_{3}\right) ; \mathrm{HR}-\mathrm{MS}$ (ESI) $m / z: \mathrm{C}_{16} \mathrm{H}_{20} \mathrm{ClNO}_{2}[\mathrm{M}+\mathrm{H}]^{+}$, Calcd. 294.1255; Found 294.1255.

(Z)-N-(4-Methylphenyl)-5-(3,3-dimethyloxiran-2-yl)-3-methylpent-2-enamide (6D), brown oil, yield 69\%. ${ }^{1} \mathrm{H}-\mathrm{NMR}\left(300 \mathrm{MHz}, \mathrm{CDCl}_{3}\right)$ 8: $7.92(\mathrm{~s}, 1 \mathrm{H}, \mathrm{NH}), 7.43(\mathrm{~d}, J=8.1 \mathrm{~Hz}, 2 \mathrm{H}, \mathrm{ArH}), 7.07(\mathrm{~d}, J=8.1 \mathrm{~Hz}, 2 \mathrm{H}$, $\mathrm{ArH}), 5.75(\mathrm{~s}, 1 \mathrm{H},=\mathrm{CH}), 2.94-2.70\left(\mathrm{~m}, 3 \mathrm{H}, \mathrm{OCH}+\mathrm{CH}_{2}\right), 2.28\left(\mathrm{~s}, 3 \mathrm{H}, \mathrm{CH}_{3}\right), 1.86(\mathrm{~d}, J=1.2 \mathrm{~Hz}, 3 \mathrm{H}$, $\left.\mathrm{CH}_{3}\right), 1.83-1.71\left(\mathrm{~m}, 2 \mathrm{H}, \mathrm{CH}_{2}\right), 1.28\left(\mathrm{~s}, 3 \mathrm{H}, \mathrm{CH}_{3}\right), 1.27\left(\mathrm{~s}, 3 \mathrm{H}, \mathrm{CH}_{3}\right)$; HR-MS (ESI) $m / z: \mathrm{C}_{17} \mathrm{H}_{23} \mathrm{NO}_{2}$ $[\mathrm{M}+\mathrm{H}]^{+}$, Calcd. 274.1802; Found 274.1798.

(Z)-N-Phenyl-5-(3,3-dimethyloxiran-2-yl)-N,3-dimethylpent-2-enamide (6E), brown oil, yield 65\%. ${ }^{1} \mathrm{H}-\mathrm{NMR}\left(300 \mathrm{MHz}, \mathrm{CDCl}_{3}\right)$ 8: 7.41-7.35 (m, 2H, ArH), 7.31-7.28 (m, 1H, ArH), 7.18-7.14 (m, 2H, $\mathrm{ArH}), 5.51(\mathrm{~s}, 1 \mathrm{H},=\mathrm{CH}), 3.31\left(\mathrm{~s}, 3 \mathrm{H}, \mathrm{NCH}_{3}\right), 2.87-2.65\left(\mathrm{~m}, 3 \mathrm{H}, \mathrm{OCH}+\mathrm{CH}_{2}\right), 1.77-1.70(\mathrm{~m}, 5 \mathrm{H}$, $\left.\mathrm{CH}_{2}+\mathrm{CH}_{3}\right), 1.32\left(\mathrm{~s}, 3 \mathrm{H}, \mathrm{CH}_{3}\right), 1.30\left(\mathrm{~s}, 3 \mathrm{H}, \mathrm{CH}_{3}\right) ; \mathrm{HR}-\mathrm{MS}$ (ESI) m/z: $\mathrm{C}_{17} \mathrm{H}_{23} \mathrm{NO}_{2}[\mathrm{M}+\mathrm{H}]^{+}, \mathrm{Calcd}$. 274.1801; Found 274.1794.

(Z)-N-Benzyl-5-(3,3-dimethyloxiran-2-yl)-3-methylpent-2-enamide (6F), yellow oil, yield 71\%. ${ }^{1} \mathrm{H}-\mathrm{NMR}$ $\left(300 \mathrm{MHz}, \mathrm{CDCl}_{3}\right)$ 8: 7.35-7.26 (m, 5H, ArH), 5.78 (br.s, $\left.1 \mathrm{H}, \mathrm{NH}\right), 5.61(\mathrm{~s}, 1 \mathrm{H},=\mathrm{CH}), 4.46(\mathrm{~d}, J=6.0 \mathrm{~Hz}$, $\left.2 \mathrm{H}, \mathrm{ArCH}_{2}\right), 2.92-2.70\left(\mathrm{~m}, 3 \mathrm{H}, \mathrm{OCH}+\mathrm{CH}_{2}\right), 1.86\left(\mathrm{~d}, J=1.2 \mathrm{~Hz}, 3 \mathrm{H}, \mathrm{CH}_{3}\right), 1.78-1.69\left(\mathrm{~m}, 2 \mathrm{H}, \mathrm{CH}_{2}\right)$, $1.28\left(\mathrm{~s}, 3 \mathrm{H}, \mathrm{CH}_{3}\right), 1.27\left(\mathrm{~s}, 3 \mathrm{H}, \mathrm{CH}_{3}\right)$; HR-MS (ESI) $m / z: \mathrm{C}_{17} \mathrm{H}_{23} \mathrm{NO}_{2}[\mathrm{M}+\mathrm{H}]^{+}$, Calcd. 274.1802; Found 274.1800 .

(Z)-N-(4-Fluorobenzyl)-5-(3,3-dimethyloxiran-2-yl)-3-methylpent-2-enamide (6G), yellow oil, yield 72\%. ${ }^{1} \mathrm{H}-\mathrm{NMR}\left(300 \mathrm{MHz}, \mathrm{CDCl}_{3}\right)$ ): 7.29-7.21 (m, 2H, ArH), 7.02-6.96 (m, 2H, ArH), 6.09 (br.s, $\left.1 \mathrm{H}, \mathrm{NH}\right)$, $5.62(\mathrm{~s}, 1 \mathrm{H},=\mathrm{CH}), 4.40\left(\mathrm{~d}, J=5.8 \mathrm{~Hz}, 2 \mathrm{H}, \mathrm{ArCH}_{2}\right), 2.93-2.66\left(\mathrm{~m}, 3 \mathrm{H}, \mathrm{OCH}+\mathrm{CH}_{2}\right), 1.85(\mathrm{~d}, J=1.2 \mathrm{~Hz}$, $\left.3 \mathrm{H}, \mathrm{CH}_{3}\right), 1.77-1.68\left(\mathrm{~m}, 2 \mathrm{H}, \mathrm{CH}_{2}\right), 1.27\left(\mathrm{~s}, 3 \mathrm{H}, \mathrm{CH}_{3}\right), 1.26\left(\mathrm{~s}, 3 \mathrm{H}, \mathrm{CH}_{3}\right)$; HR-MS (ESI) $m / z: \mathrm{C}_{17} \mathrm{H}_{22} \mathrm{FNO}_{2}$ $[\mathrm{M}+\mathrm{H}]^{+}$, Calcd. 292.1707; Found 292.1707.

(Z)-5-(3,3-Dimethyloxiran-2-yl)-3-methyl-1-morpholinopent-2-en-1-one $(6 \mathbf{H})$, yellow oil, yield 64\%. ${ }^{1} \mathrm{H}-\mathrm{NMR}\left(300 \mathrm{MHz}, \mathrm{CDCl}_{3}\right)$ \&: $5.82(\mathrm{~s}, 1 \mathrm{H},=\mathrm{CH}), 3.66\left(\mathrm{br}, 6 \mathrm{H}, 3 \times \mathrm{CH}_{2}\right), 3.51\left(\mathrm{br}, 2 \mathrm{H}, \mathrm{CH}_{2}\right), 2.76$ $(\mathrm{t}, J=6.3 \mathrm{~Hz}, 1 \mathrm{H}, \mathrm{OCH}), 2.49\left(\mathrm{t}, J=7.8 \mathrm{~Hz}, 2 \mathrm{H}, \mathrm{CH}_{2}\right), 1.87\left(\mathrm{~d}, J=1.2 \mathrm{~Hz}, 3 \mathrm{H}, \mathrm{CH}_{3}\right), 1.75-1.68(\mathrm{~m}, 2 \mathrm{H}$, $\left.\mathrm{CH}_{2}\right), 1.30\left(\mathrm{~s}, 3 \mathrm{H}, \mathrm{CH}_{3}\right), 1.28\left(\mathrm{~s}, 3 \mathrm{H}, \mathrm{CH}_{3}\right)$; HR-MS (ESI) $m / z: \mathrm{C}_{14} \mathrm{H}_{23} \mathrm{NO}_{3}[\mathrm{M}+\mathrm{H}]^{+}$, Calcd. 254.1751; Found 254.1747.

(Z)-5-(3,3-Dimethyloxiran-2-yl)-3-methyl-1-(pyrrolidin-1-yl)pent-2-en-1-one (6I), yellow oil, yield 65\%. ${ }^{1} \mathrm{H}-\mathrm{NMR}\left(300 \mathrm{MHz}, \mathrm{CDCl}_{3}\right) \delta: 5.84(\mathrm{~s}, 1 \mathrm{H},=\mathrm{CH}), 3.51\left(\mathrm{t}, J=6.6 \mathrm{~Hz}, 2 \mathrm{H}, \mathrm{NCH}_{2}\right), 3.44(\mathrm{t}, J=6.6 \mathrm{~Hz}$, $\left.2 \mathrm{H}, \mathrm{NCH}_{2}\right), 2.72(\mathrm{t}, J=6.3 \mathrm{~Hz}, 1 \mathrm{H}, \mathrm{OCH}), 2.27-2.21\left(\mathrm{~m}, 2 \mathrm{H}, \mathrm{CH}_{2}\right), 1.86\left(\mathrm{~d}, J=1.2 \mathrm{~Hz}, 3 \mathrm{H}, \mathrm{CH}_{3}\right)$, 
1.71-1.65 (m, 6H, $\left.3 \times \mathrm{CH}_{2}\right), 1.31\left(\mathrm{~s}, 3 \mathrm{H}, \mathrm{CH}_{3}\right), 1.28\left(\mathrm{~s}, 3 \mathrm{H}, \mathrm{CH}_{3}\right) ; \mathrm{HR}-\mathrm{MS}(\mathrm{ESI}) \mathrm{m} / z: \mathrm{C}_{14} \mathrm{H}_{23} \mathrm{NO}_{2}$ $[\mathrm{M}+\mathrm{H}]^{+}$, Calcd. 238.1802; Found 238.1801.

(Z)-N-Isopropyl-5-(3,3-dimethyloxiran-2-yl)-3-methylpent-2-enamide (6J), yellow oil, yield 79\%. ${ }^{1} \mathrm{H}-\mathrm{NMR}$ $\left(300 \mathrm{MHz}, \mathrm{CDCl}_{3}\right) \delta: 5.55(\mathrm{~s}, 1 \mathrm{H},=\mathrm{CH}), 5.35$ (br.s, $\left.1 \mathrm{H}, \mathrm{NH}\right), 4.14-4.06(\mathrm{~m}, 1 \mathrm{H}, \mathrm{NCH}), 2.90-2.63(\mathrm{~m}$, $\left.3 \mathrm{H}, \mathrm{OCH}+\mathrm{CH}_{2}\right), 1.84\left(\mathrm{~d}, J=1.2 \mathrm{~Hz}, 3 \mathrm{H}, \mathrm{CH}_{3}\right), 1.82-1.70\left(\mathrm{~m}, 2 \mathrm{H}, \mathrm{CH}_{2}\right), 1.38\left(\mathrm{~s}, 3 \mathrm{H}, \mathrm{CH}_{3}\right), 1.36(\mathrm{~s}, 3 \mathrm{H}$, $\left.\mathrm{CH}_{3}\right), 1.15\left(\mathrm{~d}, J=6.5 \mathrm{~Hz}, 6 \mathrm{H}, 2 \times \mathrm{CH}_{3}\right) ; \mathrm{HR}-\mathrm{MS}(\mathrm{ESI}) \mathrm{m} / z: \mathrm{C}_{13} \mathrm{H}_{24} \mathrm{NO}_{2}[\mathrm{M}+\mathrm{H}]^{+}$, Calcd. 226.1807; Found 226.1797.

\subsection{Bioassay of Fungicidal Activity}

The preliminary fungicidal activities of compounds 5-6 against F. graminearum, $R$. solani, A. solani, S. sclerotiorum, and B. cinerea were evaluated using methods in the references [33-37] by the mycelium growth rate [38]. The culture was incubated at $25 \pm 0.5^{\circ} \mathrm{C}$. Procedure for inhibition rate: The stock $2000 \mathrm{mg} / \mathrm{L}$ DMSO solution of tested compounds were prepared in advance. Then hot PDA culture medium was added into a plate, added sample solution or blank DMSO to the plate and mix with PDA culture medium, made the final concentration as desired. When plate was made, put a $5 \mathrm{~mm}$ diameter fungus cake into the center of plate, incubated them at $25 \pm 0.5^{\circ} \mathrm{C}$ for $24-48 \mathrm{~h}$, checked the growth status and calculated the inhibition rate according to the reference. Three replicates were performed and the mean measurements were calculated from the three replicates for each compound.

The $\mathrm{EC}_{50}$ values were determined from the inhibition rates of five different concentrations based on the statistics method of [39] for the compounds that had more than $70 \%$ inhibition rates. Procedure for $\mathrm{EC}_{50}$ determination: the inhibition rates of compounds against different fungus at five concentrations were evaluated as before. Toxicity regression equations were obtained by statistics analysis and the $\mathrm{EC}_{50}$ values $(\mu \mathrm{M})$ were calculated from the regression equations with excel program. Carbendazim and Chlorothalonil were used as positive control in the mycelium growth rate test.

\section{Conclusions}

(Z/E)-3,7-dimethyl-2,6-octadienamide derivatives and their 6,7-epoxy analogues were synthesized in moderate to excellent yields in four steps with the commercially available nerol/geraniol as raw materials. All the compounds were characterized by HR-ESI-MS and ${ }^{1} \mathrm{H}-\mathrm{NMR}$ spectral data. The preliminary bioassays showed that some of these compounds, such as 5C, 5 I and $\mathbf{6 b}$ exhibit $94.0 \%, 93.4 \%$ and $91.5 \%$ inhibition rates against $R$. solani at the concentration of $50 \mu \mathrm{g} / \mathrm{mL}$, respectively. The $\mathrm{EC}_{50}$ values of compounds $\mathbf{5 f}$ and $\mathbf{5 G}$ were 9.7 and $13.4 \mu \mathrm{M}$ against $R$. solani, respectively, while compound $\mathbf{5 f}$ had $\mathrm{EC}_{50}$ value of $4.3 \mu \mathrm{M}$ against $F$. graminearum. Further syntheses and structure optimization studies on the replacement of aromatic and aliphatic amines with nitrogen-containing heterocyclic amines are in progress in our laboratory.

Acknowledgments: This project was co-founded by the National Natural Science Foundation of China (No. 21172254) and the 12th Five-year National Key Technologies R \& D Program of China (No. 2011BAE06B04).

Author Contributions: M. Yang and H. Dong synthesized all of new compounds; J. Jiang ran the bioassay evaluation and statistics analysis; M. Yang drafted the paper; and M. Wang started the project, designed the molecules and revised the paper.

Conflicts of Interest: The authors declare no conflict of interest.

\section{References}

1. Antoszczak, M.; Maj, E.; Stefanska, J.; Wietrzyk, J.; Janczak, J.; Brzezinski, B.; Huczynski, A. Synthesis, antiproliferative and antibacterial activity of new amides of salinomycin. Bioorg. Med. Chem. 2014, 24, 1724-1729. [CrossRef] [PubMed]

2. Wang, X.M.; Xu, J.; Xin, M.H.; Lu, S.M.; Zhang, S.Q. Design, synthesis and antiproliferative activity evaluation of m-(4-morpholinyl-1,3,5-triazin-2-yl)benzamides in vitro. Bioorg. Med. Chem. Lett. 2015, 25, 1730-1735. [CrossRef] [PubMed] 
3. Raffa, D.; Maggio, B.; Plescia, F.; Cascioferro, S.; Raimondi, M.V.; Cancemi, G.; D'Anneo, A.; Lauricella, M.; Cusimano, M.G.; Bai, R. Synthesis, antiproliferative activity and possible mechanism of action of novel 2-acetamidobenzamides bearing the 2-phenoxy functionality. Bioorg. Med. Chem. 2015, 23, 6305-6316. [CrossRef] [PubMed]

4. Brunel, J.M.; Lieutaud, A.; Lome, V.; Pagès, J.-M.; Bolla, J.-M. Polyamino geranic derivatives as new chemosensitizers to combat antibiotic resistant Gram-negative bacteria. Bioorg. Med. Chem. 2013, 21, 1174-1179. [CrossRef] [PubMed]

5. Soni, J.N.; Soman, S.S. Synthesis and antimicrobial evaluation of amide derivatives of benzodifuran-2-carboxylic acid. Eur. J. Med. Chem. 2014, 75, 77-81. [CrossRef] [PubMed]

6. Cascioferro, S.; Cusimano, M.G.; Schillaci, D. Antiadhesion agents against Gram-positive pathogens. Future Microbiol. 2014, 9, 1209-1220. [CrossRef] [PubMed]

7. Benoit, A.R.; Schiaffo, C.; Salomon, C.E.; Goodell, J.R.; Hiasa, H.; Ferguson, D.M. Synthesis and evaluation of $\mathrm{N}$-alkyl-9-aminoacridines with antibacterial activity. Bioorg. Med. Chem. 2014, 24, 3014-3017. [CrossRef] [PubMed]

8. Zhu, S.S.; Lu, X.H.; Chen, L.; Liu, X.L. Research advances in carboxylic acid amide fungicides. J. Pestic. Sci. 2010, 12, 1-12.

9. Wang, Z.J.; Gao, Y.; Hou, Y.L.; Yu, S.J.; Bian, Q.; Li, Z.M.; Zhao, W.G. Design, synthesis, and fungicidal evaluation of a series of novel 5-methyl-1H-1,2,3-trizole-4-carboxyl amide and ester analogues. Eur. J. Med. Chem. 2014, 86, 87-94. [CrossRef] [PubMed]

10. Du, X.J.; Bian, Q.; Wang, H.X.; Yu, S.J.; Kou, J.J.; Wang, Z.P.; Li, Z.M.; Zhao, W.G. Design, synthesis, and fungicidal activity of novel carboxylic acid amides represented by $N$-benzhydryl valinamide carbamates. Org. Biomol. Chem. 2014, 12, 5427-5434. [CrossRef] [PubMed]

11. Wang, Z.J.; Liu, B.; Li, Y.H.; Zhao, W.G. Synthesis and bioactivity of novel 1-substituted-1H-1,2,3triazole-4-carboxamide. Chin. J. Org. Chem. 2011, 31, 317-323.

12. Xie, F.; Liu, T.T.; Yang, G.; Yuan, J.; Kong, X.L.; Xu, T.M.; Tan, C.X. Synthesis and acaricidal activity of new 1H-pyrazole-4-carboxamide derivatives. Chin. J. Org. Chem. 2013, 33, 2596-2601. [CrossRef]

13. Ma, Q.S.; Liu, X.H.; Weng, J.Q.; Li, Y.S.; Zhang, M.; Zhang, X.Y.; Tan, C.X. Synthesis and herbicidal activity of new pyrazine derivative. Chin. J. Org. Chem. 2013, 33, 1749-1754. [CrossRef]

14. Liu, T.T.; Ni, Y.; Zhong, L.K.; Huang, H.Y.; Hu, W.Q.; Xu, T.M.; Tan, C.X. Synthesis and fungicidal activity of difluoromethyl substituted carboxamide derivatives. Chin. J. Org. Chem. 2015, 35, 422-427. [CrossRef]

15. Liu, Y.Y.; Li, Y.; Chen, N.Q.; Lv, K.Z.; Zhou, C.; Xiong, X.H.; Li, F.S. Synthesis and fungicidal activity of novel chloro-containing 1-aryl-3-oxypyrazoles with an oximino ester or oximino amide moiety. Molecules 2014, 19, 8140-8150. [CrossRef] [PubMed]

16. Wu, W.N.; Liang, W.Q.; Sun, M.Y.; Shi, L.; Zhou, L.B.; Ouyang, G.P. Synthesis, fungicidal, and insecticide activity of novel substituted-N-(5-cyanopyridine-4-yl) benzamides. J. Chem. Pharm. Res. 2014, 6, $284-287$.

17. Yang, Y.; Jiang, J.Z.; Qimei, L.B.; Yan, X.J.; Zhao, J.X.; Yuan, H.Z.; Qin, Z.H.; Wang, M.A. The fungicidal terpenoids and essential oil from Litsea cubeba in Tibet. Molecules 2010, 15, 7075-7082. [CrossRef] [PubMed]

18. Gallardo, G.L.; Pena, N.I.; Cabrera, G.M. Neric acid derivatives produced by the honey bee fungal entomopathogen Ascosphaera apis. Phytochem. Lett. 2008, 1, 155-158. [CrossRef]

19. Dong, H.B.; Yang, M.Y.; Jiang, J.Z.; Wang, M.A. Total synthesis of 3,7-dimethyl-7-hydroxy-2-octen-1,6-olide and 3,7-dimethyl-2,6-octadien-1,6-olide. J. Asian Nat. Prod. Res. 2013, 15, 880-884. [CrossRef] [PubMed]

20. Zhao, J.; Dong, H.B.; Yang, M.Y.; Du, J.; Jiang, J.Z.; Wang, M.A. Synthesis and fungicidal activity of 7-methyl-7-hydroxy-2,3-benzo[c]octa-1,6-olide. J. Asian Nat. Prod. Res. 2014, 16, 312-317. [CrossRef] [PubMed]

21. Dong, H.B.; Yang, M.Y.; Tang, B.; Wang, M.A. Total synthesis of 3,7-dimethyl-7-hydroxy-2-octen-1,6-olide and (E)-6,7-dihydroxy-3,7-dimethyl-2-octenic acid. Chin. J. Org. Chem. 2014, 34, 2350-2353. [CrossRef]

22. Wilson, M.S.; Woo, J.C.S.; Dake, G.R. A synthetic approach toward nitiol: Construction of two 1,22-dihydroxynitianes. J. Org. Chem. 2006, 71, 4237-4245. [CrossRef] [PubMed]

23. Kim, S.; Kim, E.; Shin, D.-S.; Kang, H.; Oh, K.-B. Evaluation of morphogenic regulatory activity of farnesoic acid and its derivatives against Candida albicans Dimorphism. Bioorg. Med. Chem. Lett. 2002, 12, 895-898. [CrossRef] 
24. Thota, N.; Koul, S.; Reddy, M.V.; Sangwan, P.L.; Khan, I.A.; Kumar, A.; Raja, A.F.; Andotra, S.S.; Qazi, G.N. Citral derived amides as potent bacterial NorA efflux pump inhibitors. Bioorg. Med. Chem. 2008, 16, 6535-6543. [CrossRef] [PubMed]

25. Sureshkumar, D.; Maity, S.; Chandrasekaran, S. Regio- and stereoselective synthesis of aziridino epoxides from cyclic dienes. J. Org. Chem. 2006, 71, 1653-1657. [CrossRef] [PubMed]

26. Picquet, M.; Fernandez, A.; Bruneau, C.; Dixneuf, P.H. Efficient ruthenium-catalysed synthesis of 3-hydroxy-1-propen-1-yl benzoates: En route to an improved isomerization of 2-propyn-1-ols into $\alpha, \beta$-unsaturated aldehydes. Eur. J. Org. Chem. 2000, 13, 2361-2366. [CrossRef]

27. Velusamy, S.; Ahamed, M.; Punniyamurthy, T. Selective synthesis of $\alpha, \beta$-unsaturated ketones by dibutyltin dimethoxide-catalyzed condensation of aldehydes with alkenyl trichloroacetates. Org. Lett. 2004, 6, 4281-4283.

28. Reed, M.A.; Weaver, D.; Sun, S.; McLellan, A.; Lu, E. Terpenoid Analogues and Uses Thereof for Treating Neurological Conditions. Patent WO 2012034232 A1, 22 March 2012.

29. Zweifel, T.; Naubron, J.-V.; Grutzmacher, H. Catalyzed dehydrogenative coupling of primary alcohols with water, methanol, or amines. Angew. Chem. Int. Ed. 2009, 48, 559-563. [CrossRef] [PubMed]

30. Oakleaf, J.A.; Thomas, M.T.; Wu, A.; Snieckus, V. Alkylation of $\alpha, \beta$-unsaturated amides via metalated and dimetalated intermediates. Tetrahedron Lett. 1978, 19, 1645-1648. [CrossRef]

31. Lo, J.C.; Yabe, Y.; Baran, P.S. A Practical and catalytic olefin coupling. J. Am. Chem. Soc. 2014, 136, $1304-1307$. [CrossRef] [PubMed]

32. Chekroun, A.; Jarid, A.; Benharref, A.; Boutalib, A. Regio- and stereoselectivity of $\beta$-himachalene epoxidation by m-CPBA. A theoretical study. J. Org. Chem. 2000, 65, 4431-4434. [CrossRef] [PubMed]

33. Tang, B.; Yang, M.Y.; Zhao, Y.; Kong, L.Q.; Wang, W.W.; Wang, M.A. Discovery of 5-(5,5-Dimethylbutenolide-3-ethylidene)-2-amino-imidazolinone derivatives as fungicidal agents. Molecules 2015, 20, 13740-13752. [CrossRef] [PubMed]

34. Liu, B.; Han, J.T.; Tang, B.; Wang, M.A. Synthesis and fungicidal activity of D-Ribose and D-Xylose with hydantion. Chin. J. Org. Chem. 2014, 34, 1442-1446. [CrossRef]

35. Han, J.T.; Dong, H.B.; Xu, Z.H.; Wang, J.M.; Wang, M.A. Synthesis and activity of novel acylthiourea with hydantoin. Int. J. Mol. Sci. 2013, 14, 19526-19539. [CrossRef] [PubMed]

36. Han, J.T.; Dong, H.B.; Xu, Z.H.; Lei, J.P.; Wang, M.A. Facile synthesis of 5-arylidene thiohydantoin by sequential sulfonylation/desulfination reaction. Int. J. Mol. Sci. 2013, 14, 12484-12495. [CrossRef]

37. Lei, J.P.; Han, J.T.; Xu, Z.H.; Dong, H.B.; Wang, M.A. Synthesis and fungicidal activity of 5-cyclohexylidene2-aminoimidazolin-4-one derivatives. Chin. J. Org. Chem. 2012, 32, 1993-1998. [CrossRef]

38. Chen, N.C. The Bioassay Technologies for Pesticides; Beijing Agricultural University Press: Beijing, China, 1991; pp. 161-162.

39. Berkson, J. A statistically precise and relatively simple method of estimating the bioassay with quantal response, based on the logistic function. J. Am. Stat. Assoc. 1953, 48, 565-599. [CrossRef]

Sample Availability: Samples of the compounds $5 \mathbf{a}-\mathbf{5 N}$ and $\mathbf{6 a}-\mathbf{6 J}$ are available from the authors.

(C) 2015 by the authors; licensee MDPI, Basel, Switzerland. This article is an open access article distributed under the terms and conditions of the Creative Commons by Attribution (CC-BY) license (http://creativecommons.org/licenses/by/4.0/). 